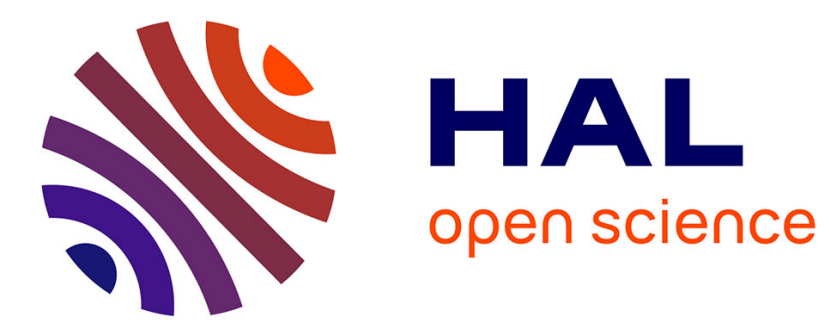

\title{
On the effect of twist angle on nonlinear galloping of suspended cables
}

\author{
Angelo Luongo, Daniele Zulli, Giuseppe Piccardo
}

\section{To cite this version:}

Angelo Luongo, Daniele Zulli, Giuseppe Piccardo. On the effect of twist angle on nonlinear galloping of suspended cables. Computers \& Structures, 2009, 87 (15-16), pp.1003-1014. hal-00788461

\section{HAL Id: hal-00788461 \\ https://hal.science/hal-00788461}

Submitted on 14 Feb 2013

HAL is a multi-disciplinary open access archive for the deposit and dissemination of scientific research documents, whether they are published or not. The documents may come from teaching and research institutions in France or abroad, or from public or private research centers.
L'archive ouverte pluridisciplinaire HAL, est destinée au dépôt et à la diffusion de documents scientifiques de niveau recherche, publiés ou non, émanant des établissements d'enseignement et de recherche français ou étrangers, des laboratoires publics ou privés. 


\title{
On the effect of twist angle on nonlinear galloping of suspended cables
}

\author{
Angelo Luongo ${ }^{a, *}$, Daniele Zulli ${ }^{a}$, Giuseppe Piccardo ${ }^{b}$ \\ a DISAT - University of L'Aquila, 67040 L'Aquila, Italy \\ ${ }^{\mathrm{b}}$ DICAT - University of Genoa, 16145 Genova, Italy
}

\begin{abstract}
A B S T R A C T
A nonlinear model of cable, able to twist, is formulated. For small sag to length ratios (e.g. 1/10) and technical parameter values proper to electrical transmission lines, the motion is ruled by the classical equations of the perfectly flexible cable, plus a further equation governing the twist evolution. A two degree of freedom system is successively obtained via a Galerkin procedure. The relevant nonlinear ODE's are dealt with a Multiple Scale approach, under 2:1 internal resonance condition and no resonance conditions, in order to investigate Hopf bifurcations and post critical behaviors. All the numerical results are compared with those furnished by the flexible model, and the influence of twist is discussed.
\end{abstract}

\section{Introduction}

It is well known that the aerodynamic forces acting on a non circular rigid cylinder, subjected to a wind flow, depend, besides on the mean wind velocity, on the exposure to the flow of the body, that is on the attitude of the cylinder cross section. When an elastic beam is analyzed in the framework of the quasi steady theory of the aerodynamic forces, the loads are usually evaluated referring to the initial attitude of the section (see [1]), and rota tions taken into account only to determine, in an approximate manner (see [2]), the fluid to structure relative velocity. A more refined analysis, however, is possible, in which the time depen dent actual attitude of the cross section is considered, as de scribed by the so called twist angle (i.e. by the rotation of the section around its normal axis). In contrast, when a pretensioned string is studied, such an angle is usually not included among the kinematic descriptors of the body, since all the rotations are believed to be unimportant in capturing the main structural behavior. Therefore, a model of perfectly flexible string is adopted (one dimensional not polar continuum), and the aerodynamic forces evaluated with reference to the initial attitude of the sec tion, which is assumed to remain immutable in time. The prob lem is made even more complicated when a sagged cable is considered. Indeed, due to the steady part of the aerodynamic forces and to the high flexibility of the structure, the cable signif icantly changes its equilibrium configuration and, therefore, its

\footnotetext{
* Corresponding author.

E-mail addresses: luongo@ing.univaq.it (A. Luongo), danzulli@ing.univaq.it (D. Zulli), giuseppe.piccardo@unige.it (G. Piccardo).
}

exposure to the flow. Hence, in addition to a dynamic rotation, a static velocity dependent rotation of the section must be con sidered in evaluating the aerodynamic forces.

The aeroelastic instability of sagged cables has been widely studied in the literature. Luongo and Piccardo [3] have studied the nonlinear galloping of cables in 2:1 internal resonance condi tion, by using a perfectly flexible cable model $[4,5]$ and account ing for the static rotation only. In a successive work [6], they have tentatively corrected the classical cable model to account for the twist, by using a quite simplified model. Yu et al. [7], McConnel and Chang [8], White et al. [9] have employed a model of cable beam, accounting for twisting but not for bending, and neglecting the cable initial curvature in defining the torsion strain. In contrast, they have considered a realistically coupled extension torsion constitutive law, based on experimental re sults. Recently, Luongo et al. [10], have formulated a consistent linear model of cable beam accounting for the (small) curvature of the cable, as well as for bending and torsional stiffness. By retaining only the leading terms in each equations, they obtained linear reduced equations, amenable to an analytical solution, identical to that of the perfectly flexible model, plus an additional equation accounting for both bending and torcent moments. The model permitted to detect the influence of the dynamic twist on the critical wind velocity.

In this paper, the model presented in [10] is reformulated in the nonlinear range and nonlinear, reduced equations are derived along the same lines. These equations are obviously a particular case of more complete models, as, for example, that of Lu and Per kins [11]; these models, however, are composed by very complex equations and suffer of some numerical problems related to the 
existence of boundary layers, caused by the smallness of the flex ural terms (nearly singular equations). A simple two degree of freedom nonlinear system is then derived from the continuous model via a Galerkin procedure, and the critical and post critical aeroelastic behavior of the cable investigated in resonant and non resonant cases via a Multiple Scale perturbation approach (see [12]). The role played by the dynamic twist is finally highlighted.

The paper is organized as follows. The reduced equations of mo tion are formulated in Section 2. The discretization is performed in Section 3. The perturbation analysis is carried out in Section 4, where the amplitude modulation equations are derived. These lat ter are numerically studied in Section 5 for some sample systems. Finally, some conclusions are drawn in Section 6.

\section{Model}

The cable is modeled as a body made of a flexible centerline and rigid cross sections restrained to remain orthogonal to the axis (shear undeformable beam). It is assumed to be uniformly iced and loaded by a wind flow of mean velocity $\mathbf{U} \quad U \mathbf{a}_{z}$, blowing hor izontally. Three different configurations are considered, described in the following (Fig. 1). (a) The initial configuration $\mathscr{C}_{0}$, taken by the body at the time $t \quad 0$, under the action of its self weight $m g \mathbf{a}_{y}$ (including the ice accretion). This configuration is planar, and belongs to the vertical $\left(\mathbf{a}_{x}, \mathbf{a}_{y}\right)$ plane. The cable is prestressed in $\mathscr{C}_{0}$ by an axial internal force $T_{0}\left(s_{0}\right)$, depending on the (un stretched) abscissa $s_{0}$; other internal forces (shear, bending and torcent moments) are neglected. (b) The reference configuration $\overline{\mathscr{C}}$, assumed by the body at the time $t \quad 0^{+}$, in which static wind forces $\overline{\mathbf{b}}_{a}(s)$ (with the stretched abscissa $s \simeq s_{0}$ ) act on the cable. Under the simplifying hypothesis that $\overline{\mathbf{b}}_{a}$ is uniform on the length of the cable, $\overline{\mathscr{C}}$ still lies in a plane, forming an angle $\varphi$ with the vertical plane. If shear and internal moments are still neglected, equilib rium requires that the resultant force $\overline{\mathbf{b}}: \overline{\mathbf{b}}_{a} \quad m g \mathbf{a}_{y}$ lies in the plane of the cable. By vanishing its component along the binormal direction $\overline{\mathbf{a}}_{3}$, it follows that:

$b_{a_{3}}(\varphi, U)+m g \sin \varphi \quad 0$

being $\bar{b}_{a_{3}} \quad \overline{\mathbf{b}}_{a} \quad \overline{\mathbf{a}}_{3}, \mathbf{a}_{y} \quad \overline{\mathbf{a}}_{3} \quad \sin \varphi$, and where the dependence on the static aerodynamic force $\bar{b}_{a_{3}}$ on the configuration variable $\varphi$ and on the flow velocity $U$ has been made explicit. Eq. (1) implicitly defines the nonlinear, non trivial equilibrium path $\varphi \quad \varphi(U)$. In this wind dependent reference configuration $\overline{\mathscr{C}}$, the cable is prestressed by an axial internal force $\bar{T}(s)$, modified with respect to $\mathscr{C}_{0}$ by the static aerodynamic forces. Due to this circumstance, also the natural frequencies and modes of the cable are modified. (c) The actual con figuration $\mathscr{C}$, assumed by the cable at the time $t>0$, in which the body is loaded also by (non uniform) dynamic wind forces $\mathbf{b}_{a} \quad \overline{\mathbf{b}}_{a}$, depending on displacement and velocity of the cable. In this

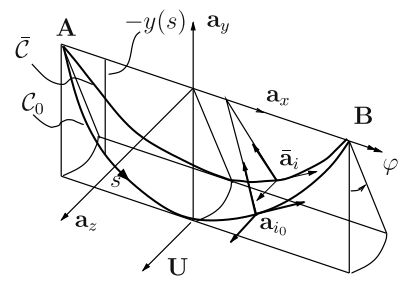

(a)

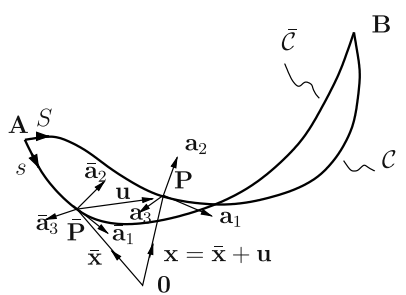

(b)
Fig. 1. Cable configurations: (a) initial $\mathscr{C}_{0}$ and reference $\overline{\mathscr{C}}$ configurations; (b) actual configuration $\mathscr{C}$. configuration $\mathscr{C}$, all the internal forces and moments are considered to contribute to the dynamic equilibrium of the body.

The equations of motion governing the dynamics of the cable, referred to the configuration $\overline{\mathscr{C}}$, are derived in the following. The mechanical model and the aerodynamic model are formulated separately.

\subsection{Mechanical model}

The reference configuration $\overline{\mathscr{C}}$ is described by the planar curve $\overline{\mathbf{x}} \quad \overline{\mathbf{x}}(s)$ and by the cross section inertial principal triad $\overline{\boldsymbol{\beta}}: \quad\left\{\overline{\mathbf{a}}_{1}(s, t), \overline{\mathbf{a}}_{2}(s, t), \overline{\mathbf{a}}_{3}(s, t)\right\}$, assumed to be coincident with the Frenet triad (Fig. 1b). Here, $\overline{\mathbf{a}}_{1} \equiv \overline{\mathbf{x}}^{\prime}$ is the tangent, $\overline{\mathbf{a}}_{2}$ the normal and $\overline{\mathbf{a}}_{3}$ the binormal to the curve, the dash denoting $s$ differentia tion. Therefore, $\overline{\mathbf{a}}_{1}^{\prime} \quad \bar{\kappa} \overline{\mathbf{a}}_{2}, \overline{\mathbf{a}}_{2}^{\prime} \quad \bar{\kappa} \overline{\mathbf{a}}_{1}, \overline{\mathbf{a}}_{3}^{\prime} \quad \mathbf{0}$, with $\bar{\kappa} \quad \bar{\kappa}(s)$ the cur vature in $\overline{\mathscr{C}}$.

The actual configuration of the body is described by the non planar curve $\mathbf{x} \mathbf{x}(s, t)$ and the inertial principal triad $\boldsymbol{\beta}$ : $\left\{\mathbf{a}_{1}(s, t), \mathbf{a}_{2}(s, t), \mathbf{a}_{3}(s, t)\right\}$.

The transport is described by the displacement vector field $\mathbf{u}(s, t)$ and the rotation tensorial field $\mathbf{R}(s, t)$, which leads the triad $\overline{\boldsymbol{\beta}}$ to match the triad $\boldsymbol{\beta}$ :

$\mathbf{x} \mathbf{x}+\mathbf{u}, \quad \mathbf{a}_{i} \quad \mathbf{R a}_{i}, \quad i \quad 1,2,3$.

The scalar representation of $\mathbf{R}$, involving three elementary rotations $\vartheta_{i}$, is given in [13] and in many other papers. The shear undeformabil ity constraints require that, in the actual configuration, the tangent $\mathbf{x}^{\prime}$ to the centerline is parallel to the normal $\mathbf{a}_{1}$ with regard to the cross section, namely, $\mathbf{x}^{\prime} \quad(1+\varepsilon) \mathbf{a}_{1}$, where $\varepsilon$ is the axial strain. From this condition, the strain $\varepsilon$ and the rotations $\vartheta_{2}$ and $\vartheta_{3}$ are derived as func tions of four independent configuration variables, the three compo nents $u, v$ and $w$ of vector $\mathbf{u}$ on $\overline{\boldsymbol{\beta}}$, and the twist angle $\vartheta: \vartheta_{1}$. Then, the incremental bendings $\hat{\kappa}_{2}, \hat{\kappa}_{3}$ and torsion $\hat{\kappa}_{1}$ are introduced as inde pendent components on $\overline{\boldsymbol{\beta}}$ of the skew symmetric tensor:

\section{$\widehat{\mathbf{K}} \quad \mathbf{R}^{\mathrm{T}} \mathbf{R}^{\prime}$.}

The following strain measures, expanded up to second order terms, are obtained [13]:

$$
\begin{aligned}
& \varepsilon \quad u^{\prime} \quad \kappa v+\frac{1}{2}\left[\left(v^{\prime}+\kappa u\right)^{2}+w^{\prime}\right], \\
& \hat{\kappa}_{1} \quad \vartheta^{\prime}+\kappa w^{\prime}+\kappa^{2} v w^{\prime}+w^{\prime} v^{\prime \prime}+\kappa^{\prime} u w^{\prime}, \\
& \left.\hat{\kappa}_{2} \quad w^{\prime \prime}+\kappa \vartheta+\left[\begin{array}{ll}
u^{\prime} & \kappa v
\end{array}\right) w^{\prime}\right]^{\prime}+\vartheta\left[(\kappa u)^{\prime}+v^{\prime \prime}\right], \\
& \hat{\kappa}_{3} \quad v^{\prime \prime}+(\kappa u)^{\prime}+\vartheta w^{\prime \prime} \quad \frac{1}{2} \kappa\left(\vartheta^{2}+w^{\prime 2}\right) \quad\left[\left(\kappa u+v^{\prime}\right)\left(\begin{array}{ll}
u^{\prime} & \kappa v
\end{array}\right]^{\prime} .\right.
\end{aligned}
$$

The equations of motion are derived via the extended Hamilton principle (see, e.g. [14]):

$$
\begin{aligned}
& \delta H: \quad \int_{t_{1}}^{t_{2}} \int_{0}^{\ell}\left\{m(\dot{u} \delta \dot{u}+\dot{v} \delta \dot{v}+\dot{w} \delta \dot{w})+\mathscr{J}_{1} \dot{\vartheta} \delta \dot{\vartheta}+\left(b_{1} \quad c_{u} \dot{u}\right) \delta u\right. \\
& +\left(\begin{array}{ll}
b_{2} & c_{v} \dot{v}
\end{array}\right) \delta v+\left(\begin{array}{ll}
b_{3} & c_{w} \dot{w}
\end{array}\right) \delta w+\left(\begin{array}{ll}
c_{1} & c_{\vartheta} \dot{\vartheta}
\end{array}\right) \delta \vartheta+E A \varepsilon \delta \varepsilon \\
& \left.+G J \hat{\kappa}_{1} \delta \hat{\kappa}_{1}+E I_{2} \hat{\kappa}_{2} \delta \hat{\kappa}_{2}+E I_{3} \hat{\kappa}_{3} \delta \hat{\kappa}_{3} \quad \bar{T}_{\varepsilon_{I I}} \delta \varepsilon_{\mathrm{II}}\right\} \mathrm{d} s \mathrm{~d} t \quad 0 \\
& \forall \delta u, \delta v, \delta w, \delta \vartheta \text {, }
\end{aligned}
$$

where $\ell$ is the cable length; $E A, G J, E I_{2}$ and $E I_{3}$ are the axial, torsional and bending stiffnesses, respectively; $b_{1}, b_{2}, b_{3}$ and $c_{1}$ are the exter nal forces and couple densities; $c_{u}, c_{v}, c_{w}$ and $c_{\vartheta}$ are the structural damping coefficients; $\varepsilon_{\text {II }}$ is the second order part of the axial strain, accounting for the prestress working; $m$ is the mass linear density and $I_{1}$ is the inertia polar moment of the section. It should be noted that an uncoupled constitutive law among the incremental internal forces and the incremental strains has been assumed in Eq. (5). By substituting Eq. (4) in Eq. (5), performing the variations and inte grating by parts, a set of four differential equations in the indepen dent configuration variables is drawn. By enforcing the constraint 
conditions $\delta u \quad \delta v \quad \delta w \quad 0$ and $\delta \vartheta$ arbitrary at the ends (spherical hinges), the boundary conditions also follow from the variational principle. Identical equations were obtained in [13], where a differ ent approach, based on direct equilibrium and later condensation of the shear forces, was followed.

The boundary value problem so far obtained is not reported here, since the equations are too complicated. They, however, as sume a much simpler form if a order of magnitude analysis is per formed along the lines already followed in [10] for the associated linear problem, and only the leading terms are retained in each equation. The analysis is based on the following assumptions: the nondimensional parameter $\delta: 8 d / \ell$, expressing the sag to length ratio, is small; consequently, $\bar{\kappa}(s) \simeq$ const, $\bar{T}(s) \simeq$ const; the nondimensional prestress $\tau: \bar{T} / E A$ is small of order $\delta^{3}$; the nondimensional characteristic inertia radius $\varrho \quad r / \ell$ of the section is also small of order $\delta^{3}$; the nondimensional stiffness parameter $\beta$ : GJ/EI is of order 1 ; the transversal displacements $v$ and $w$ are of the same order, while $\mathcal{O}(u / v) \quad \delta$ and $\mathcal{O}(\vartheta d / w) \quad 1$; the translations vary on a typical scale of length $\ell$ (since they vanish at the ends) while the twist varies on a much greater length (since it does not vanish at the ends). The following reduced equations are thus obtained:

$$
\begin{aligned}
& E A\left[u^{\prime} \quad \kappa v+\frac{1}{2} v^{\prime 2}+\frac{1}{2} w^{\prime 2}\right]^{\prime}+b_{1} \quad m \ddot{u} \quad c_{u} \dot{u} \quad 0,
\end{aligned}
$$

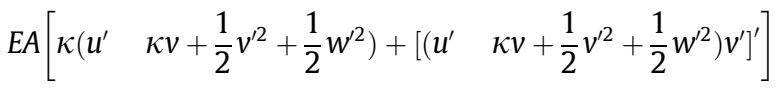

$$
\begin{aligned}
& +\bar{T} v^{\prime \prime}+b_{2} \quad m \ddot{v} \quad c_{v} \dot{v} \quad 0,
\end{aligned}
$$

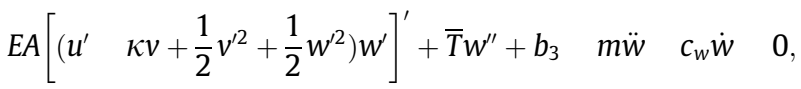

$$
\begin{aligned}
& G J \vartheta^{\prime \prime} \quad E I \kappa^{2} \vartheta+(E I+G J) \kappa w^{\prime \prime} \quad E I \kappa \vartheta v^{\prime \prime}+G J\left(v^{\prime \prime} w^{\prime}\right)^{\prime} \\
& +c_{1} \quad \mathscr{J}_{1} \ddot{\vartheta} \quad c_{\vartheta} \dot{\vartheta} \quad 0
\end{aligned}
$$

with the relevant boundary conditions:

$$
u \quad v \quad w \quad 0, G J\left(\vartheta^{\prime}+\kappa w^{\prime}+w^{\prime} v^{\prime \prime}\right) \quad 0, \quad \text { at } s \quad 0, \ell \text {. }
$$

As a major result of the analysis, and in perfect analogy with the lin ear problem [10], Eqs. (6) and (7) show that, at the leading order, the dynamics of the cable are governed by the classical equations of the perfectly flexible model (Eq. (6) $)_{a-c}$, identical to that of Lee and Perkins [5] and usually adopted in the literature) plus an addi tional Eq. $(6)_{d}$, governing the twist around the tangent. It is interest ing to note that, while the bending does not affect the translational dynamics, it, in contrast, contributes to the twist dynamics, differ ently from certain models used in the literature [7 9], where it is inconsistently neglected.

To better appreciate the influence of bending on twist dynam ics, it is instructive to derive Eq. $(6)_{\mathrm{d}}$ in an alternative way. Let us consider the moment equilibrium equation around the tangent to the cable in the actual configuration:

$M_{1}^{\prime} \quad M_{2} \kappa_{3}+M_{3} \kappa_{2}+c_{1} \quad 0$,

where $M_{i} \quad \mathbf{m} \mathbf{a}_{i} ; c_{1} \quad \mathbf{c} \mathbf{a}_{1} ; \kappa_{2} \quad \hat{\kappa}_{2}$ and $\kappa_{3} \quad \bar{\kappa}+\hat{\kappa}_{3}$ are the ac tual curvature components. If this equation is linearized, then $\mathbf{a}_{i} \simeq \overline{\mathbf{a}}_{i}, \kappa_{2} \simeq 0$ and $\kappa_{3} \simeq \bar{\kappa}$; moreover, if reduced expressions for the curvatures are considered, consistently with the approxima tions introduced:

$\hat{\kappa}_{1} \simeq \vartheta^{\prime}+\kappa w^{\prime}+u^{\prime} v^{\prime \prime}, \quad \hat{\kappa}_{2} \simeq w^{\prime \prime}+\kappa \vartheta+\vartheta v^{\prime \prime}$

and the constitutive laws $M_{1} \quad G J \hat{\kappa}_{1}, M_{2} \quad E I_{2} \hat{\kappa}_{2}$ are used, Eq. (6) $)_{\mathrm{d}}$ is finally recovered. The procedure shows that neglecting bending in the twist equation leads to inconsistent equilibrium violation, since the ignored terms are of the same order of that retained in the analysis.
Eqs. (6) and (7) can be further simplified if the assumption of quasi steady stretching and twisting is introduced. It is based on the fact that the transversal to longitudinal and transversal to tor sional squared frequency ratios are small. Therefore, inertia and damping forces can be neglected in Eqs. (6) $)_{a, d}$ and the tangential displacement $u$ and the twist angle $\vartheta$ determined in integral form, this latter via a perturbation method (see Appendix A). The follow ing expressions are found, holding for $b_{1} \quad 0, c_{1} \quad 0$ :

$$
\begin{aligned}
u(s, t) & \frac{s}{\ell} \int_{0}^{\ell}\left(\begin{array}{lll}
\kappa v & \frac{1}{2} v^{\prime 2} & \frac{1}{2} w^{\prime 2}
\end{array}\right) \mathrm{d} s \\
+ & \int_{0}^{s}\left[\begin{array}{lll}
\kappa v(\xi, t) & \frac{1}{2} v^{\prime}(\xi, t)^{2} & \frac{1}{2} w^{\prime}(\xi, t)^{2}
\end{array}\right] \mathrm{d} \xi
\end{aligned}
$$

and

$$
\begin{aligned}
& \vartheta \quad\left(A_{1}+A_{2}\right) \cosh k s+\left(B_{1}+B_{2}\right) \sinh k s \quad \frac{G J+E I}{\sqrt{ } G J E I} \int_{0}^{s} w^{\prime \prime}(\xi, t) \\
& \times \sinh [k(s \quad \xi)] \mathrm{d} \xi \quad \frac{1}{k} \int_{0}^{s}\left(v^{\prime \prime}(\xi, t) w^{\prime}(\xi, t)\right)^{\prime} \sinh [k(s \quad \xi)] \mathrm{d} \xi \\
& +\sqrt{\frac{E I}{G J}} \int_{0}^{s} v^{\prime \prime}(\zeta, t)\left(\frac{G J+E I}{\sqrt{ } G J E I} \int_{0}^{\zeta} w^{\prime \prime}(\xi, t) \sinh [k(\zeta \quad \xi)] \mathrm{d} \xi\right. \\
& \left.\left.+A_{1} \cosh k \zeta+B_{1} \sinh k \zeta\right) \sinh \left[\begin{array}{ll}
k & \zeta
\end{array}\right)\right] \mathrm{d} \zeta,
\end{aligned}
$$

where $k: \quad \bar{\kappa} \sqrt{ } E I / G J$ and $A_{1}, B_{1}, A_{2}, B_{2}$ are determined by the bound ary conditions.

By taking into account Eqs. (10) and (11), the problem is gov erned by two integro differential equation (Eq. $(6)_{b, c}$ ) in the sole unknowns $v(s, t)$ and $w(s, t)$. However, in view of a later discretiza tion of the field equations (Section 3 ), it results more convenient, from a computational point of view, do not integrate the twist equation $(6)_{d}$ (i.e. do not use the cumbersome Eq. (11)), but rather to append its static counterpart to the integro differential equa tions. Therefore, the problem is formulated as

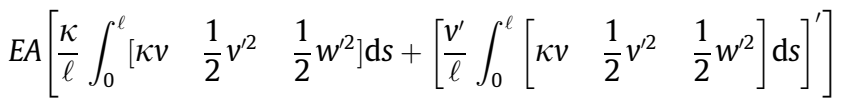

$$
\begin{aligned}
& +\bar{T} v^{\prime \prime}+b_{2} \quad m \ddot{v} \quad c_{v} \dot{v} \quad 0,
\end{aligned}
$$

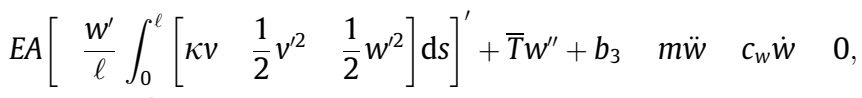

$$
\begin{aligned}
& G J \vartheta^{\prime \prime} \quad E I \kappa^{2} \vartheta+(E I+G J) \kappa w^{\prime \prime} \quad E I \kappa \vartheta v^{\prime \prime}+G J\left(v^{\prime \prime} w^{\prime}\right)^{\prime} \quad 0 .
\end{aligned}
$$

\subsection{Aerodynamic model}

A simple aerodynamic model is adopted here, based on the fol lowing simplifying assumptions: (a) the quasi steady theory [2] is adopted; (b) the curvature of the cable is neglected; (c) loads are evaluated taking into account the twist angle, but neglecting the (smaller) flexural rotations; (d) the ice is uniformly distributed along the cable; (e) the aerodynamic couples are neglected. Fig. 2 shows the attitudes of the cross section in the initial configuration (axes $\mathbf{a}_{2_{0}}, \mathbf{a}_{3_{0}}$ ), in the reference configuration (axes $\overline{\mathbf{a}}_{2}, \overline{\mathbf{a}}_{3}$, rotated by $\varphi$ ) and in the actual configuration (axes $\mathbf{a}_{2}, \mathbf{a}_{3}$, still further rotated by $\vartheta$ ). The static rotation $\varphi$ only depends on the mean wind veloc ity via Eq. (1); the dynamic rotation $\vartheta$ also depends on the abscissa $s$ and the time $t$. The angle $\varphi$ is assumed to be large, the angle $\vartheta$ small but finite.

According to the quasi steady theory, the flow exerts on the section the aerodynamic force:

$\mathbf{b}_{a} \quad \frac{1}{2} \rho_{a} \operatorname{Vr}\left(c_{\mathrm{d}}(\gamma) \mathbf{V}+c_{\mathrm{l}}(\gamma) \mathbf{a}_{1} \times \mathbf{V}\right)$, 


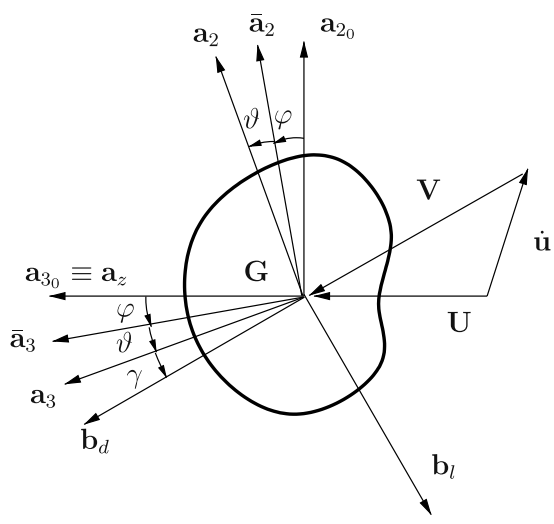

Fig. 2. Attitudes of the cross-section ( $\gamma$ angle of attack, $\mathbf{b}_{\mathrm{d}}$ and $\mathbf{b}_{1}$ drag and lift forces, respectively).

where $\rho_{a}$ is the air density, $r$ is a characteristic dimension of the cable cross section, $\mathbf{V} \quad U \quad \dot{v} \overline{\mathbf{a}}_{2} \quad \dot{w} \overline{\mathbf{a}}_{3}$ is the relative velocity of the wind with respect to the center $\mathbf{G}$ of the section, $V\|\mathbf{V}\|$ its modulus, and $c_{\mathrm{d}}$ and $c_{1}$ two aerodynamic coefficients, called of drag and lift, respectively. These latter depend on the shape of the section and on the angle of attack

$\gamma: \arcsin \left(\frac{\mathbf{V}}{V} \cdot \mathbf{a}_{2}\right)$,

i.e. on the angle between $\mathbf{V}$ and a reference material axis, here taken as $\mathbf{a}_{3}$. The two components of $\mathbf{b}_{a}$, along wind $\mathbf{b}_{\mathrm{d}}$ and cross wind $\mathbf{b}_{1}$, are usually known as drag and lift forces, respectively (Fig. 2). In Eq. (13) the relative velocity $\mathbf{V}$ was evaluated ignoring the effect of the twist velocity $\dot{\vartheta}$. This, indeed, usually accounted for in the literature through the rather questionable concept of characteristic radius $r_{\mathrm{c}}$ [2], is negligible in the problem at hand, since it entails velocities at the boundary of the section of the order of $\dot{\vartheta} r_{c}$, which are smaller than the velocity $\dot{w}$ of the centerline, being $\dot{\vartheta} \quad \mathcal{O}(\dot{w} / d)$. In contrast, the twist $\vartheta$ does affect the aerodynamic forces via the attack angle $\gamma$; consequently, $\mathbf{b}_{a} \quad \mathbf{b}_{a}(\vartheta, \dot{v}, \dot{w} ; \varphi(U), U)$.

By substituting Eq. (14) in Eq. (13), expanding for small $\vartheta$, $\dot{v}$ and $\dot{w}$ up to cubic terms, and projecting on the $\overline{\mathbf{a}}_{2}, \overline{\mathbf{a}}_{3}$ axes, the follow ing force components are derived:

$b_{a_{i}} \quad b_{a_{i}}(\varphi)+\sum_{j 1}^{3} c_{i j}(\varphi) \xi_{j}+\sum_{j, k 1}^{3} c_{i j k}(\varphi) \xi_{j} \xi_{k}+\sum_{j, k, l}^{3} c_{i j k l}(\varphi) \xi_{j} \xi_{k} \xi_{1}$,

i 2,3

where $\xi$ : $\quad(\vartheta, \dot{v}, \dot{w})^{\mathrm{T}}$ is the vector collecting the independent vari ables, $\bar{b}_{a_{i}}$ are the static forces, and $c_{i j}, c_{i j k}$ and $c_{i j k l}$ are coefficients depending on $c_{\mathrm{d}}, c_{\mathrm{l}}$ and their derivatives with respect to $\gamma$, all eval uated at $\mathscr{\mathscr { C }}$. The coefficients, which assume quite involved expres sions, are reported in Appendix B.

\section{Discrete model}

A discrete model is drawn by Eq. (12) via a Galerkin procedure. The displacement field is discretized as follows:

$$
\left(\begin{array}{c}
\vartheta(s, t) \\
v(s, t) \\
w(s, t)
\end{array}\right) \quad \sum_{j=1}^{m}\left(\begin{array}{c}
0 \\
\phi_{v_{j}} \\
0
\end{array}\right) q_{j}^{i}+\sum_{k 1}^{n}\left(\begin{array}{c}
\phi_{\vartheta_{k}} \\
0 \\
\phi_{w_{k}}
\end{array}\right) q_{k}^{o}
$$

where $q_{j}^{i}(t), j \quad 1, \ldots, m$, are the unknown amplitudes of the in plane trial functions $\phi_{v_{j}}$, and $q_{k}^{o}(t), k 1, \ldots, n$, are the unknown amplitudes of the out of plane trial functions $\phi_{w_{k}}, \phi_{\vartheta_{k}}$. The transla tional modes are deduced from the associated linearized Hamilto nian problem $\left(c_{i j} \quad 0,[15]\right)$ whereas the torsional mode is derived from the later Eq. (35) $)_{a}$ (Appendix A). In order to obtain a suffi ciently simple nonlinear model, a sole in plane, $\phi_{v}(s)$, and a sole out of plane, $\left\{\phi_{w}(s), \phi_{\vartheta}(s)\right\}$, eigenfunction ( $m \quad n \quad 1$ in Eq. (16)) are selected. By taking into account the expression (15) of aerody namic forces, two ordinary differential equations are obtained:

$$
\begin{aligned}
\ddot{q}_{2} & +2 \zeta_{2} \omega_{2} \dot{q}_{2}+\omega_{2}^{2} q_{2}+h_{23} q_{3}+c_{a 22} \dot{q}_{2}+c_{a 23} \dot{q}_{3}+h_{1} q_{2}^{2} \\
& +h_{2} q_{3}^{2}+h_{3} q_{2} q_{3}^{2}+h_{4} q_{2}^{3}+h_{5} q_{3}^{3}+d_{1} q_{3} \dot{q}_{2}+d_{2} q_{3} \dot{q}_{3} \\
& +d_{3} \dot{q}_{2}^{2}+d_{4} \dot{q}_{2} \dot{q}_{3}+d_{5} \dot{q}_{3}^{2}+d_{6} q_{3}^{2} \dot{q}_{2}+d_{7} q_{3}^{2} \dot{q}_{3}+d_{8} q_{3} \dot{q}_{2}^{2} \\
& +d_{9} q_{3} \dot{q}_{2} \dot{q}_{3}+d_{10} q_{3} \dot{q}_{3}^{2}+d_{11} \dot{q}_{2}^{3}+d_{12} \dot{q}_{2}^{2} \dot{q}_{3} \\
& +d_{13} \dot{q}_{2} \dot{q}_{3}^{2}+d_{14} \dot{q}_{3}^{3} \quad 0, \\
\ddot{q}_{3} & +2 \zeta_{3} \omega_{3} \dot{q}_{3}+\omega_{3}^{2} q_{3}+h_{33} q_{3}+c_{a 32} \dot{q}_{2}+c_{a 33} \dot{q}_{3}+h_{6} q_{2} q_{3} \\
& +h_{7} q_{3}^{2}+h_{8} q_{2}^{2} q_{3}+h_{9} q_{3}^{3}+d_{15} q_{3} \dot{q}_{2}+d_{16} q_{3} \dot{q}_{3}+d_{17} \dot{q}_{2}^{2} \\
& +d_{18} \dot{q}_{2} \dot{q}_{3}+d_{19} \dot{q}_{3}^{2}+d_{20} q_{3}^{2} \dot{q}_{2}+d_{21} q_{3}^{2} \dot{q}_{3}+d_{22} q_{3} \dot{q}_{2}^{2}+d_{23} q_{3} \dot{q}_{2} \dot{q}_{3} \\
& +d_{24} q_{3} \dot{q}_{3}^{2}+d_{25} \dot{q}_{2}^{3}+d_{26} \dot{q}_{2}^{2} \dot{q}_{3}+d_{27} \dot{q}_{2} \dot{q}_{3}^{2}+d_{28} \dot{q}_{3}^{3} \quad 0,
\end{aligned}
$$

where $q_{2}(t)$ and $q_{3}(t)$ describe the in plane and out of plane time laws, respectively; $\omega_{2}$ and $\omega_{3}$ are the in plane and out of plane nat ural frequencies of the cable; the $c_{a}$ 's are the aerodynamic damping coefficients; the coefficients $\zeta_{2}$ and $\zeta_{3}$ are structural damping ratios assumed to be of proportional type. Both quadratic and cubic non linearities appear in the equations of motion. The twist $\vartheta$ leads, in particular, to the appearance of a $2 \times 2$ circulatory matrix H $\left[0, h_{23} ; 0, h_{33}\right]$, and of terms mixed in velocity displacement; moreover, it also contributes to some nonlinear mechanical coeffi cients. All the coefficients of Eq. (17) are defined in Appendix C. The effect of the mean wind velocity is implicit in Eq. (17), since it is included in the static angle of rotation $\varphi$, from which, in turn, all the coefficients of the equations depend on through the refer ence axial tension $\bar{T}$ or the aerodynamic coefficients.

\section{Amplitude modulation equations}

The Multiple Scale perturbation method (MSM) is employed to attack Eq. (17). As a first hypothesis, let us assume that in plane and out of plane natural frequencies are different, $\omega_{2} \neq \omega_{3}$, i.e. the cable has a small but finite sag. Then, a dimensionless pertur bation parameter $\epsilon$ is introduced $\left(\epsilon \ll 1\right.$ ), and the unknowns $q_{2}, q_{3}$ are expanded in series of $\epsilon$ :

$q_{k} \quad \epsilon q_{k 1}+\epsilon^{2} q_{k 2}+\epsilon^{3} q_{k 3}, \quad k \quad 2,3$.

Two independent slow time scales, $t_{1}$ and $t_{2}$, are introduced, in addition to the fast scale $t_{0}\left(t_{n} \quad \epsilon^{n} t, n \quad 0,1,2\right)$, so that the first and second time derivatives are expressed as $\mathrm{d} / \mathrm{d} t \quad d_{0}+\epsilon d_{1}+$ $\epsilon^{2} d_{2}+$, and $\mathrm{d}^{2} / \mathrm{d} t^{2} d_{0}^{2}+2 \epsilon d_{0} d_{1}+\epsilon^{2}\left(d_{1}^{2}+2 d_{0} d_{2}\right)+$, where $d_{n} \partial / \partial t_{n}$. Moreover, it is assumed that the linear dissipative forces depending on velocity (both mechanical and aerodynamic damp ing) are small of order $\epsilon$, while the coefficients of the circulatory matrix $\mathbf{H}$ (depending on $U^{2}$, see Appendices $C$ and $B$ ) are of order 1 , since they can become comparable to the stiffnesses when the mean wind velocity takes sufficiently high values. The $\epsilon$ order per turbation equations are thus obtained:

$$
\left(\begin{array}{l}
d_{0}^{2} q_{21} \\
d_{0}^{2} q_{31}
\end{array}\right)+\left[\begin{array}{cc}
\omega_{2}^{2} & h_{23} \\
0 & \omega_{3}^{3}
\end{array}\right]\left(\begin{array}{l}
q_{21} \\
q_{31}
\end{array}\right) \quad\left(\begin{array}{l}
0 \\
0
\end{array}\right)
$$

being $\bar{\omega}_{3} \quad \sqrt{ } \omega_{3}^{2}+h_{33}$; from Eq. (19) the following generating solu tions come out:

$$
\left(\begin{array}{l}
q_{21} \\
q_{31}
\end{array}\right) \quad A_{2}\left(t_{1}, t_{2}\right)\left(\begin{array}{l}
1 \\
0
\end{array}\right) \mathrm{e}^{\mathrm{i} \omega_{2} t_{0}}+A_{3}\left(t_{1}, t_{2}\right)\left(\begin{array}{c}
\frac{h_{23}}{\omega_{2}^{2} \omega_{3}^{2}} \\
1
\end{array}\right) \mathrm{e}^{\mathrm{i} \omega_{3} t_{0}}+\text { c.c. },
$$

where $\mathrm{i}$ is the imaginary unit, $A_{2}, A_{3}$ are the complex amplitudes, which are unknowns functions of the slow times, and the term 
c.c. denotes the complex conjugate. It should be noted that the cir culatory terms $h_{i j}$ strongly affect the generating solution (20), both directly and through an alteration of the out of plane natural fre quency $\omega_{3}$. In particular, since the matrix of the generating problem (19) is not symmetric, it is necessary to find its right and left eigen vectors (Appendix D), these latter necessary to impose the solvabil ity conditions at the higher orders.

At this point, in order to obtain the high order perturbation equations, one needs to distinguish between non resonant and internal resonant conditions (of $m: 1$ type, with $m \neq 1$ ). For a sagged cable two cases are usually of interest: (a) 2:1 internal resonance, that is the in plane natural frequency is about twice the out of plane natural frequency; (b) no internal resonance condition.

\subsection{Internal resonance condition}

The particular condition of 2:1 internal resonance occurs when the cable is close to the first cross over point [15], i.e.:

$\omega_{2} \quad 2 \omega_{3}+\epsilon \sigma, \quad \sigma \quad \mathcal{O}(1)$

$\sigma$ being a detuning parameter. Using the generating solution (20), the following $\epsilon^{k}$ order perturbation equations are obtained:

$$
\begin{aligned}
& \left(\begin{array}{l}
d_{0}^{2} q_{2 k} \\
d_{0}^{2} q_{3 k}
\end{array}\right)+\left[\begin{array}{cc}
\omega_{2}^{2} & h_{23} \\
0 & \omega_{3}^{3}
\end{array}\right]\left(\begin{array}{l}
q_{2 k} \\
q_{3 k}
\end{array}\right) \quad \mathbf{t}_{k 2} \mathrm{e}^{\mathrm{i} \omega_{2} t_{0}}+\mathbf{t}_{k 3} \mathrm{e}^{\mathrm{i} \omega_{3} t_{0}}+\mathrm{NST}, \\
& \quad k \quad 2,3
\end{aligned}
$$

where $\mathbf{t}_{k 2}, \mathbf{t}_{k 3}$ are two vectors collecting coefficients that multiply the resonant exponential, $\mathrm{e}^{\mathrm{i} \omega_{2} t_{0}}$ and $\mathrm{e}^{\mathrm{i} \omega_{3} t_{0}}$, respectively, in the two equations; NST denotes not secular terms. The solvability condition requires that the resonant vectors $\mathbf{t}_{k 2}, \mathbf{t}_{k 3}$ are orthogonal to the left eigenvectors (Appendix D), $\mathbf{v}_{2} \mathbf{t}_{k 2} \quad 0, \mathbf{v}_{3} \mathbf{t}_{k 3} \quad 0$, leading to equa tions of the type:

$$
\begin{array}{lll}
d_{1} A_{2} & \mathfrak{I}_{12}\left(A_{2}, A_{3}^{2} ; U\right), \quad d_{2} A_{2} & \mathfrak{I}_{22}\left(A_{2}, A_{3}^{2}, A_{2} A_{3} \bar{A}_{3}, A_{2}^{2} \bar{A}_{2} ; U\right), \\
d_{1} A_{3} & \mathfrak{I}_{13}\left(A_{3}, \bar{A}_{3} A_{2} ; U\right), \quad d_{2} A_{3} & \mathfrak{I}_{23}\left(A_{3}, \bar{A}_{3} A_{2}, A_{2} \bar{A}_{2} A_{3}, A_{3}^{2} \bar{A}_{3} ; U\right) .
\end{array}
$$

Eq. (23) govern the amplitude modulation on the slow temporal scales. In order to return to the time $t$ one needs to apply the recon stitution rule $\dot{A}_{k} \quad \epsilon d_{1} A_{k}+\epsilon^{2} d_{2} A_{k}, k \quad 2,3$, and then to reabsorb the perturbation parameter in the coefficients, obtaining the (complex) amplitude equations:

$$
\begin{array}{ll}
\dot{A}_{2} & \mathfrak{I}_{2}\left(A_{2}, A_{3}^{2}, A_{2} A_{3} \bar{A}_{3}, A_{2}^{2} \bar{A}_{2} ; U\right), \\
\dot{A}_{3} & \mathfrak{I}_{3}\left(A_{3}, \bar{A}_{3} A_{2}, A_{2} \bar{A}_{2} A_{3}, A_{3}^{2} \bar{A}_{3} ; U\right)
\end{array}
$$

being $\mathfrak{I}_{k} \quad \mathfrak{J}_{1 k}+\mathfrak{J}_{2 k}$. They can be written in real form adopting the polar representation $A_{k} \quad 1 / 2 a_{k} \mathrm{e}^{\mathrm{i} \alpha_{k}, k} \quad 2,3$, being $a_{k}$ the real ampli tude and $\alpha_{k}$ the phase of $A_{k}$, respectively. By separating Eq. (24) into real and imaginary parts, four state equations in the real variables $a_{k}$ and $\alpha_{k}$ are obtained. These equations can be transformed into an autonomous system by letting:

$$
\begin{array}{lll}
\delta & \alpha_{2} & 2 \alpha_{3}+\sigma t
\end{array}
$$

The result is a set of three equations called amplitude modulation equations (AME):

$$
\begin{aligned}
& \dot{a}_{2} \quad a_{2} p_{10}+\frac{1}{2} a_{3}^{2}\left(p_{21}^{*} \cos \delta+p_{22}^{*} \sin \delta\right)+\frac{1}{4} a_{2} a_{3}^{2} p_{31}+\frac{1}{4} a_{2}^{3} p_{41}, \\
& \dot{a}_{3} \quad a_{3}\left[p_{50}+\frac{1}{2} a_{2}\left(p_{61}^{*} \cos \delta \quad p_{62}^{*} \sin \delta\right)+\frac{1}{4} a_{2}^{2} p_{71}+\frac{1}{4} a_{3}^{2} p_{81}\right], \\
& a_{2} a_{3} \dot{\delta} \quad a_{3}\left[\begin{array}{lll}
a_{2}\left(p_{12}\right. & \left.2 p_{52}+\sigma\right) & a_{2}^{2}\left(p_{61}^{*} \sin \delta+p_{62}^{*} \cos \delta\right)
\end{array}\right. \\
& +\frac{a_{3}^{2}}{2}\left(p_{21}^{*} \sin \delta+p_{22}^{*} \cos \delta\right)+\frac{a_{2}^{3}}{4}\left(p_{42} \quad 2 p_{72}\right) \\
& \left.+\frac{a_{2} a_{3}^{2}}{4}\left(p_{32} \quad 2 p_{82}\right)\right]
\end{aligned}
$$

where the coefficients $p$ are functions of the mean wind velocity $U$. They are defined in Appendix E with regards to the linear terms, which furnishes an analytical approximation of the eigenvalues. All the symbolic manipulation, indispensable to obtain the cumber some expressions of nonlinear $p$ coefficients, are performed through the software Mathematica ${ }^{\circledR}$ [16], with the help of advice in cluded in Nayfeh and Chin [17]. The fixed points $\left(a_{2}, a_{3}, \delta\right)$ of the dynamical system (26) correspond to limit cycles of the original dis crete system (17).

The AME (26) are formally identical to those obtained in Luongo and Piccardo [3] for large static displacement, where the effect of the dynamic twist $\vartheta$ was not taken into account. On the other hand, as already observed, the dynamic twist is able to deeply modify the generating solutions, then the quantitative values of all the coeffi cients $p$. Anyway, the qualitative properties of the solutions are maintained and, from the analysis of Eq. (26), the existence of the following branches of fixed points is proved ([3], see Fig. 3): branch I, $a_{2} a_{3} \quad 0, \delta$ arbitrary, $\forall U$; branch II, $a_{3} 0, a_{2}$ $a_{2}(U) \quad \sqrt{ } 4 p_{10} / p_{41}, \delta$ arbitrary; branch III, $a_{3} \quad a_{3}(U), a_{2} a_{2}(U)$, $\delta \quad \delta(U)$.

Studying the bifurcation of branch I $\left(a_{2} \rightarrow 0, a_{3} \rightarrow 0, U \rightarrow U_{\mathrm{cr}}\right)$ Eq. (26) reduce to

$a_{2} p_{10}^{\mathrm{cr}} \quad 0$

$a_{3} p_{50}^{\mathrm{cr}} \quad 0$,

$a_{2} a_{3}\left(p_{12}^{\mathrm{cr}} \quad 2 p_{52}^{\mathrm{cr}}+\sigma\right) \quad 0$,

where $p_{i}^{\mathrm{cr}} p_{i}\left(U_{\mathrm{cr}}\right)$, being $U_{\mathrm{cr}}$ the critical mean wind velocity at which the bifurcation occurs. Two different solutions of Eq. (27) can exist: $a_{2}$ bifurcation, $a_{3} \quad 0, a_{2} \neq 0$ when $p_{10}^{\text {cr }} \quad 0 ; a_{3}$ bifurcation, $a_{2} \quad 0, a_{3} \neq 0$ when $p_{50}^{\mathrm{cr}} \quad 0$. In the first case the branch bifurcates in $a_{2}$ direction (branch II in Fig. 3), while, in the second case, it bifur cates in $a_{3}$ direction. However, if the static rotation of cable is ne glected $(\varphi \quad 0)$, the coefficient $p_{50}$ is always negative for value of $U$ of technical interest because of the physics of the problem (the drag coefficient $c_{\mathrm{d}}$ is positive for any kind of cross section); there fore, critical conditions in $a_{3}$ direction can occur for large mean wind velocity only (i.e. large static rotation $\varphi$ ), not considered here. Anyhow, galloping is mono modal in both the cases, since only one mode is triggered at bifurcation. In the sequel of the paper the name "branch II" $\left(\left(a_{2}, a_{3}\right) \quad\left(a_{2}(U), 0\right)\right)$ denotes a curve bifurcated in the $\left(a_{2}, U\right)$ plane; its particular shape (with a first bifurcation at $B_{1}$ and a return to stability at $B_{2}$ ) is essentially due to the effect of the mean wind (i.e. the static rotation $\varphi$ ), whose rise produces a

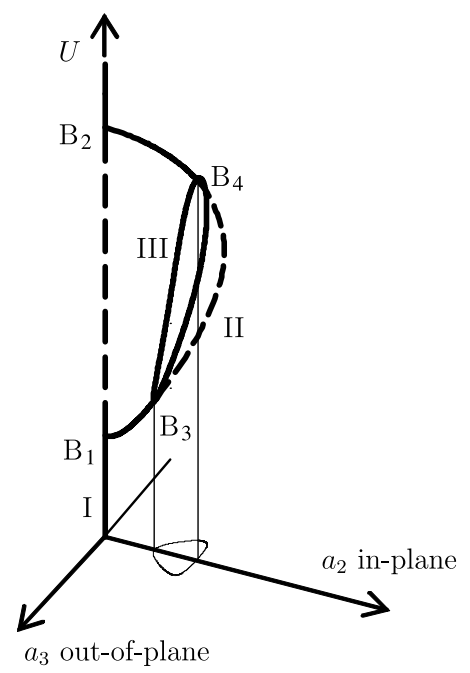

Fig. 3. Steady-state amplitude solutions vs. mean wind velocity: qualitative behavior. 
decreasing of the self excited aerodynamic forces [18]. In order to have a bi modal galloping (branch III), one needs to analyze possi ble bifurcations of branch II, looking for the existence of two com ponent solutions $\left(a_{3} \neq 0, a_{2} \neq 0\right)$ close to the branch. Bi modal galloping can occur for a sufficiently high value of in plane oscilla tions [18] as a consequence of the nonlinear interaction between the two internally resonant modes, mainly governed by quadratic mechanical terms.

\subsection{No internal resonance condition}

Let us consider a suspended cable with a small but finite sag, so that its natural frequencies are sufficiently well separated, $\omega_{2} \neq \omega_{3}$. Using the generating solution (20), $\epsilon^{k}$ order perturbation equations are found, having a structure formally similar to Eq. (22). The solv ability condition leads to equations of the type:

$$
\begin{array}{llll}
d_{1} A_{2} & \widehat{\mathfrak{I}}_{12}\left(A_{2} ; U\right), & d_{2} A_{2} & \widehat{\mathfrak{I}}_{22}\left(A_{2}, A_{2} A_{3} \bar{A}_{3}, A_{2}^{2} \bar{A}_{2} ; U\right), \\
d_{1} A_{3} & \widehat{\mathfrak{I}}_{13}\left(A_{3} ; U\right), & d_{2} A_{3} & \widehat{\mathfrak{I}}_{23}\left(A_{3}, A_{2} \bar{A}_{2} A_{3}, A_{3}^{2} \bar{A}_{3} ; U\right),
\end{array}
$$

that are simpler than the corresponding resonant Eq. (23). By apply ing the reconstitution rule $\dot{A}_{k} \epsilon d_{1} A_{k}+\epsilon^{2} d_{2} A_{k}, k 2,3$ and reab sorbing the perturbation parameter, one obtains the (complex) amplitude equations:

$$
\begin{array}{ll}
\dot{A}_{2} & \widehat{\mathfrak{I}}_{2}\left(A_{2}, A_{2} A_{3} \bar{A}_{3}, A_{2}^{2} \bar{A}_{2} ; U\right), \\
\dot{A}_{3} & \widehat{\mathfrak{I}}_{3}\left(A_{3}, A_{2} \bar{A}_{2} A_{3}, A_{3}^{2} \bar{A}_{3} ; U\right)
\end{array}
$$

being $\widehat{\mathfrak{I}}_{k} \quad \widehat{\mathfrak{J}}_{1 k}+\widehat{\mathfrak{J}}_{2 k}$. Similarly to the resonant case, they can be written in real form adopting the polar representation and separat ing real and imaginary parts:

$$
\begin{array}{ll}
\dot{a}_{2} & a_{2} p_{10}+\frac{1}{4} a_{2} a_{3}^{2} \hat{p}_{31}+\frac{1}{4} a_{2}^{3} p_{41}, \\
\dot{a}_{3} & a_{3} p_{50}+\frac{1}{4} a_{2}^{2} a_{3} \hat{p}_{71}+\frac{1}{4} a_{3}^{3} \hat{p}_{81}, \\
\dot{\alpha}_{2} & p_{12}+\frac{1}{4} a_{3}^{2} \hat{p}_{32}+\frac{1}{4} a_{2}^{2} p_{42}, \\
\dot{\alpha}_{3} & p_{52}+\frac{1}{4} a_{2}^{2} \hat{p}_{72}+\frac{1}{4} a_{2}^{2} \hat{p}_{82},
\end{array}
$$

where the hat remembers that the expression of these coefficients is not coincident to the resonant case. Eq. (30) represents the non resonance AME, in which the four equations are two by two uncoupled; then, the fixed points are determined by the first two Eq. (30), directly. Moreover, the coefficients p's related to linear amplitudes are equal to those of the resonant AME (26) (Appendix E). This occurrence implies that the internal resonance doesn't af fect the critical conditions of the system (i.e. the bifurcation points) but only its post critical behavior.

The discussion of the AME (30) is initially similar to the reso nant case. In particular, the branch I $\left(\begin{array}{lll}a_{2} & a_{3} & 0, \forall U)\end{array}\right)$ and the branch II $\left(a_{3} \quad 0, a_{2} \quad a_{2}(U) \quad \sqrt{ } 4 p_{10} / p_{41}\right)$ have the same expres sion of branches determined in internal resonance conditions. Dif ferences subsist concerning the possible existence of a bi modal galloping defined by the branch III, $a_{3} \quad a_{3}(U), a_{2} \quad a_{2}(U)$. A non resonant bi modal branch can arise from the mono modal branch II or in an independent way. In the first case, the existence of a two component solutions is analyzed solving the limit of equa tions $(30)_{\mathrm{a}}$ and $(30)_{\mathrm{b}}$ for $a_{2} \rightarrow a_{2_{0}}, a_{3} \rightarrow 0, \quad U \rightarrow U_{0}$, being $a_{2_{0}} \quad a_{2}\left(U_{0}\right)$ and marking with the index 0 a possible bifurcation point on the mono modal branch II. The existence of this kind of branch III results possible only if $p_{50}^{0}+a_{2_{0}}^{2} p_{71}^{0} / 4 \quad 0$. The second case happens observing that, in the fixed point analysis, Eqs. $(30)_{\mathrm{a}}$ and $(30)_{\mathrm{b}}$ can be simplified in the following linear form: $\begin{array}{ll}x_{2} p_{41}+x_{3} \hat{p}_{31} & 4 p_{10} \\ x_{2} \hat{p}_{71}+x_{3} \hat{p}_{81} & 4 p_{50}\end{array}$

being $x_{2} \quad a_{2}^{2}$ and $x_{3} \quad a_{3}^{2}$. The solutions of Eq. (31) can be easily studied using the well known Kramer's rule. Therefore, the exis tence of this kind of branch III is possible only when the three deter minants deriving from the linear problem have all the same sign (since $x_{2}, x_{3}$ must be positive). In both these cases the existence of branch III seems more unlikely for non resonant cases because of the absence of internal resonance conditions.

\section{Numerical results}

In order to numerically illustrate the proposed theory, the mechanical and aerodynamic cable properties are selected, to gether with the eigenfunctions to be used in the discrete model. Then, the critical conditions are analyzed with particular attention to the influence of the twist angle and to the accuracy of the per turbation solutions. Finally, the post critical equilibrium patterns are investigated, pointing out the alterations due to the torsional effects.

\subsection{Cable properties and static response}

The mechanical and aerodynamic properties of the sample cable was taken from the literature [7,3]. Concerning the mechan ical aspects, it is assumed that the axial stiffness $E A$ is $29.7 \times 10^{6} \mathrm{~N}$, the torsional stiffness $G J$ is $159 \mathrm{~N} \mathrm{~m}^{2}$, the cable diameter is $0.0281 \mathrm{~m}$, the cable length $\ell$ is about $267 \mathrm{~m}$, the sag $d$ is $6.18 \mathrm{~m}$ and the damping ratio coefficients $\zeta$ are equal to $0.44 \%$ (setting $\left.\begin{array}{lll}\zeta_{2} & \zeta_{3} & \zeta\end{array}\right)$; moreover, a bending stiffness EI $2100 \mathrm{~N} \mathrm{~m}^{2}$ is as sumed, in accord with experimental observations of sufficiently tensioned cables [19]. Therefore, the sample cable is initially close to the first cross over point [15], with an internal resonance condi tion of 2:1 type. It is easy to verify that the squared ratio between the transversal frequency $\omega_{3}$ and the torsional frequency $\omega_{\vartheta}$ is small, justifying the assumption of quasi steady twisting [10].

As regards the aerodynamic properties, two different $U$ shaped conductors are taken into account, always referring to the litera ture: a first cross section with the symmetry axis placed on $\mathbf{a}_{z}$ direction (CS1 in the sequel, Fig. 4a), having its maximum ice eccentricity opposite to the mean wind $(m \quad 1.80 \mathrm{~kg} / \mathrm{m}$ ice included [7]), and a second cross section with the symmetry axis rotated of $44.4^{\circ}$ with respect to $\mathbf{a}_{z}$ direction (CS2 in the sequel), having greater ice thickness ( $m \quad 2.0 \mathrm{~kg} / \mathrm{m}$ ice included [20]). In both the cases the specified configuration is the most prone to instability, as it happens in the usual galloping analysis. On the contrary, in the proposed theory, this really occurs in no wind conditions only, since the attitude of the section to wind, and a

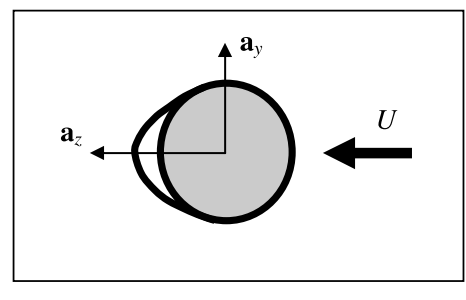

Fig. 4. (a) U-shaped conductor; (b) attitude of cross-section to wind ( $\varphi$ positive anticlockwise). b

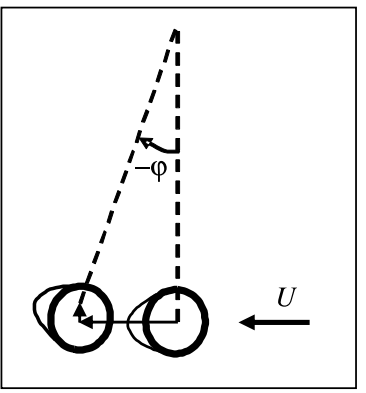


therefore, its aerodynamic coefficients, statically vary through the angle of rotation $\varphi$ (Fig. 4b). Therefore, when galloping actually oc curs, the cable cross section is generally rotated with respect to the most dangerous configuration.

Concerning the selection of discrete modes that have to be used in the analysis, it was proved in [10], both theoretically and numer ically, that the dynamic twist is more important in symmetric modes than in antisymmetric ones. Therefore, in order to investi gate the maximum effect of twist, the first symmetric in plane and out of plane modes are considered in the discrete model.

Fig. 5 shows the nonlinear equilibrium path $\varphi \quad \varphi(U)$ (Fig. 5a) for the two different cross sections previously described, and the modifications of the reference axial tension $\bar{T}$ owing to static wind loads (Fig. 5b); differences in the graphs are imputable to dissimi lar aerodynamic coefficients and mass per unit length. It should be noted that, when the mean wind velocity increases, the static rota tion $\varphi$ soon achieves large values, and the pre stress $\bar{T}$ also under goes non negligible changes.

\subsection{Critical conditions}

The conditions of incipient instability are examined by evaluat ing the real part of the two couples of complex conjugate eigen values $\lambda$ of the discrete system. For small $U, \mathfrak{R}(\lambda)<0$, for all $\lambda$ 's, so that the reference configuration $\overline{\mathscr{C}}$ is stable. At a critical velocity $U_{\mathrm{c}}$ a couple of complex conjugate eigenvalues, having maximum real part, crosses the imaginary axis, i.e. $\max \mathfrak{R}(\lambda) \quad 0$. This occur rence causes loss of stability of the equilibrium through a Hopf bifurcation, from which a limit cycle arises. The objective of this analysis is to highlight the role of the dynamic twist angle $\vartheta$ on the critical wind velocity $U_{\mathrm{c}}$. The eigenvalues can be computed either by numerical and perturbation algorithms. The numerical computation calls for solving the linear eigenvalue problem associ ated with the linearized equations (17); the AME (26) and (30) fur nish asymptotic expressions for the real part of the eigenvalues, namely $p_{10} \equiv \mathfrak{R}\left(\lambda_{2}\right)$ and $p_{50} \equiv \mathfrak{R}\left(\lambda_{3}\right)$, being $\lambda_{2}$ and $\lambda_{3}$ the in plane and out of plane eigenvalue, respectively. Examples here pre sented were previously investigated in Luongo et al. [10] by using a standard numerical approach. They are now reconsidered and analyzed through the perturbation solutions, consistently with the developed theory. Results are then compared.

Fig. 6 shows the real part of eigenvalues for both the sections described in the previous Section, taking into account (continuous lines) or neglecting (dashed lines) the circulatory matrix $\mathbf{H}$; the numerical solutions (triangular points) are also reported as regards the complete model. About CS1 (Fig. 6a), differences are limited to the second bifurcation $B_{2}$ of the in plane eigenvalues $\lambda_{2}$, where the stability of the planar equilibrium configuration is regained; the curves related to the stable eigenvalue $\lambda_{3}$ are all coincident. Con cerning CS2 (Fig. 6b), the twist angle has again quantitatively small influence on the critical eigenvalue values, $\lambda_{2}$, but it is decisive from a qualitative point of view, since the occurrence or not of both bifurcations depends on it. In both these examples the perturba tion solutions are practically coincident with the numerical ones.

To magnify the influence of dynamic twist on critical condi tions, the basic examples must be modified in their mechanical and aerodynamic properties (Fig. 7). Concerning CS1 (Fig. 7a), the basic case has been modified with a reduction of sag (that exalts the role of $\vartheta$; Luongo et al. [10]) and an initial rotation of the cross section (e.g. $1^{\circ}$ ); in this way, the cable cross section reaches the most dangerous attitude for galloping instability closer to the first bifurcation point $B_{1}$. Moreover, an increase of damping is con sidered in order to have changes in the first bifurcation values as well. Remarkable differences are found between the two different models (Fig. 7a); in any case the perturbation approximation is excellent. The influence of dynamic twist appears still more evi dent if a cross section initially non symmetric, like CS2, is consid ered (Fig. 7b). Decreasing the sag and considering an initial rotation of $47^{\circ}$ (instead of the basic value of $44^{\circ}$ ), large altera tions of the critical wind velocities are found. The perturbation solution is able to capture the critical points with a good approxi mation, even if quantitative errors appear in the critical eigenvalue locus.

\subsection{Post critical behavior}

The complete nonlinear AME (26) and (30) are now used to investigate the effective influence of the twist on the nonlinear range. Steady state solutions of the AME (limit cycle amplitudes) are considered, both for resonant and non resonant cables.

First, the internal resonance case is discussed. The sag of the cable is fixed to the value $d \quad 6.18 \mathrm{~m}$, which corresponds to a detuning $\sigma+0.0093 \mathrm{rad} / \mathrm{s}$ for CS1 and $\sigma \quad 0.0876 \mathrm{rad} / \mathrm{s}$ for CS2 in no wind conditions (this perceptible difference is due to dif ferent values of cable mass per unit length). Fig. 8 shows the mod al amplitudes for CS1. The basic case (Fig. 8a) admits mono modal galloping only, with appreciable differences due to the dynamic twist. No bi modal galloping occurs since the in plane amplitude remains comparatively small. If, in contrast, the cable cross section is rotated (i.e. it is no more symmetric) in no wind conditions (Fig. 8b), the first bifurcation occurs when the aerodynamic coeffi cients are closer to the most dangerous values, so that branch III takes place. Differences produced by the twist angle are still appre ciable. Fig. 9 shows the modal amplitudes for CS2. The basic sample
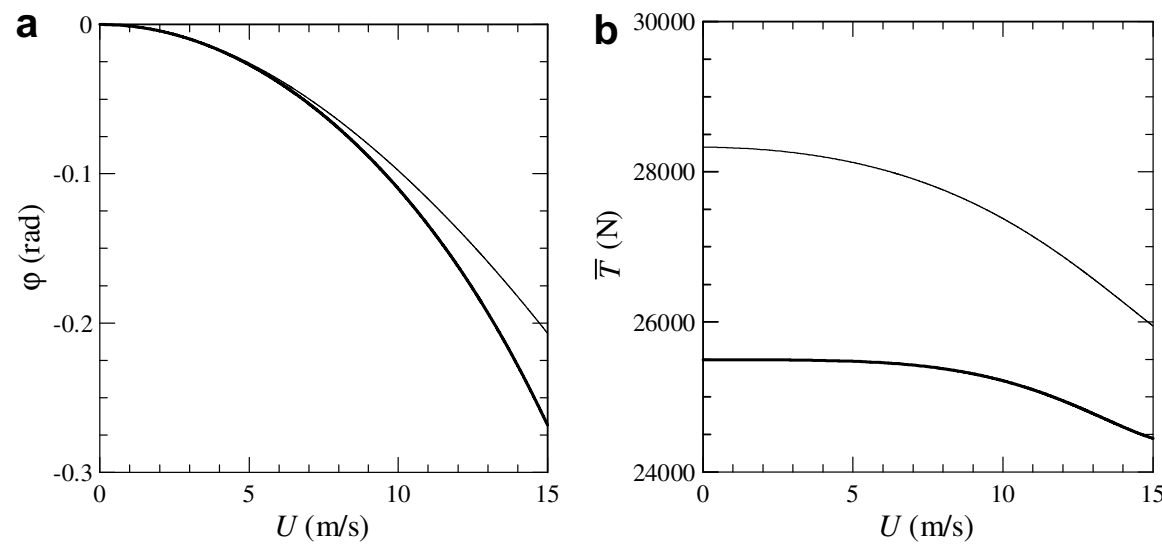

Fig. 5. (a) Nonlinear equilibrium path; (b) prestress (thick lines: CS1, thin lines CS2). 

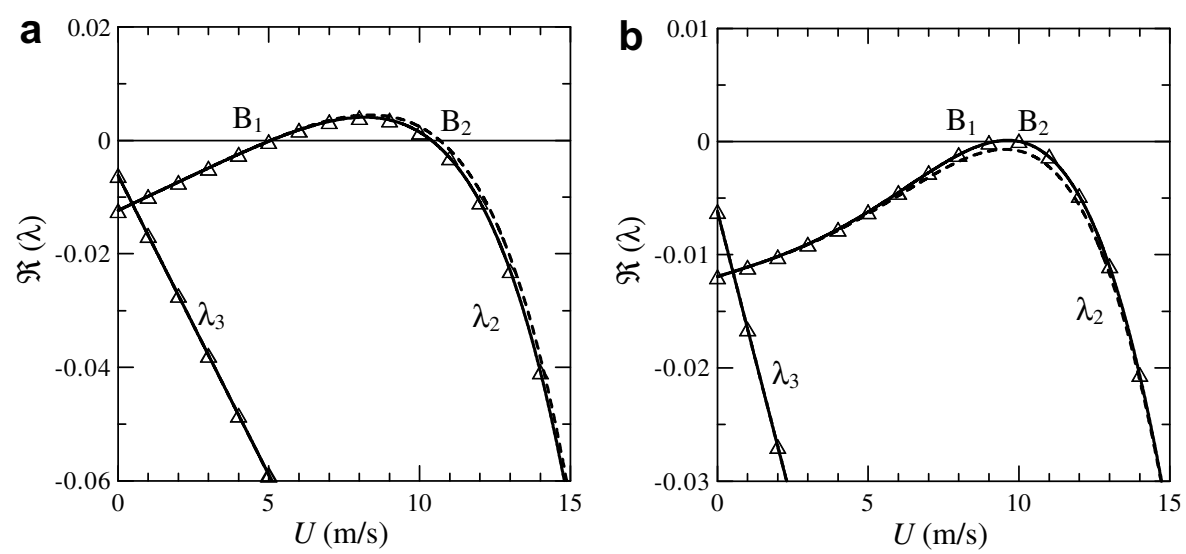

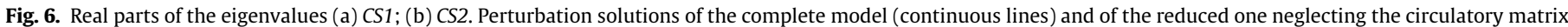
(dashed lines); $\triangle$ : numerical solutions.
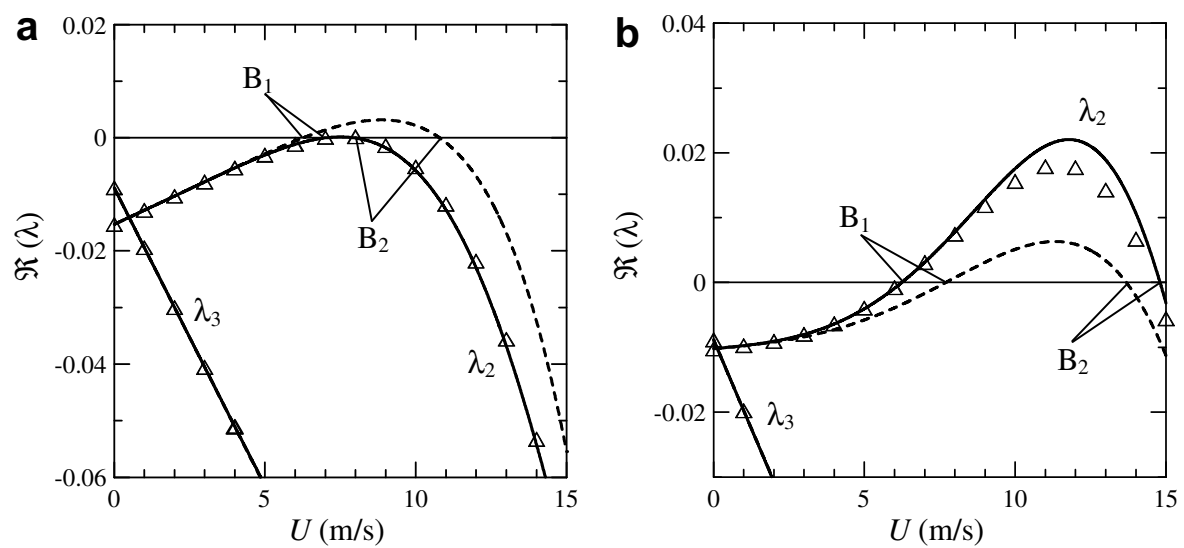

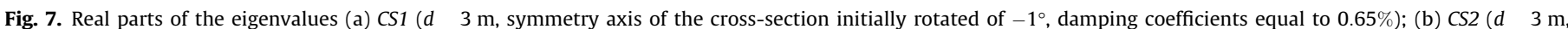

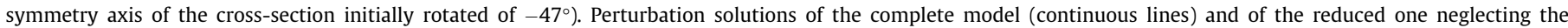
circulatory matrix (dashed lines); $\Delta$ : numerical solutions.
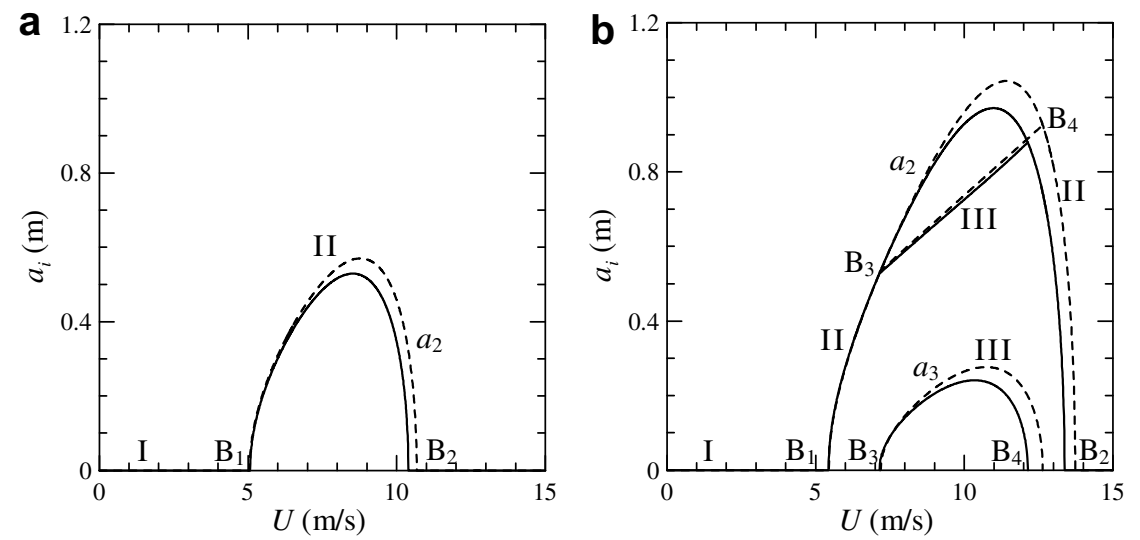

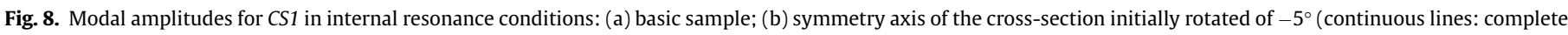
model; dashed lines: no dynamic twist angle effect).

(Fig. 9a) admits a mono modal galloping (branch II) only when the dynamic twist angle is considered; in any case, this path is con fined in a narrow region, both of velocity and amplitude. To obtain more relevant equilibrium patterns, it is necessary to reduce the detuning and slightly to increase the sag (thus enhancing the mod al coupling); moreover, it needs to start with a section considerably rotated with respect to the basic sample (thus moving the post critical branches towards higher mean velocities). Fig. 9b shows the (restricted) bi modal galloping obtained, highlighting changes due to the dynamic twist angle. It should be noted the greater dif ficulty in obtaining branch III, compared to the analyses by Luongo and Piccardo [3] where the sole effect of the static twist was 

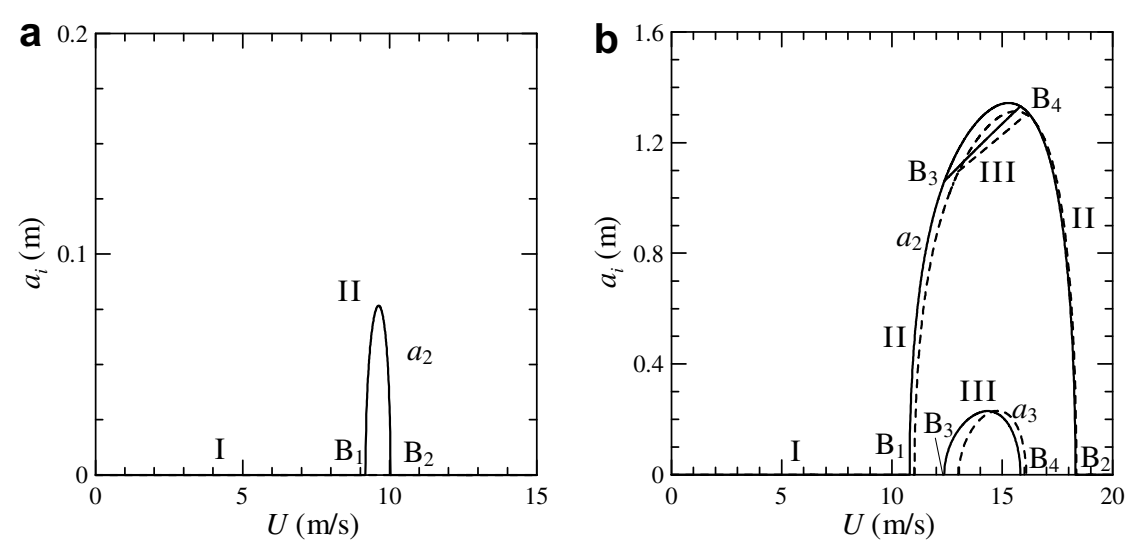

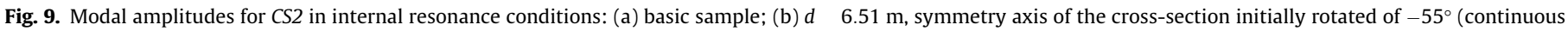
lines: complete model; dashed lines: no dynamic twist angle effect). The scale of ordinates is different.
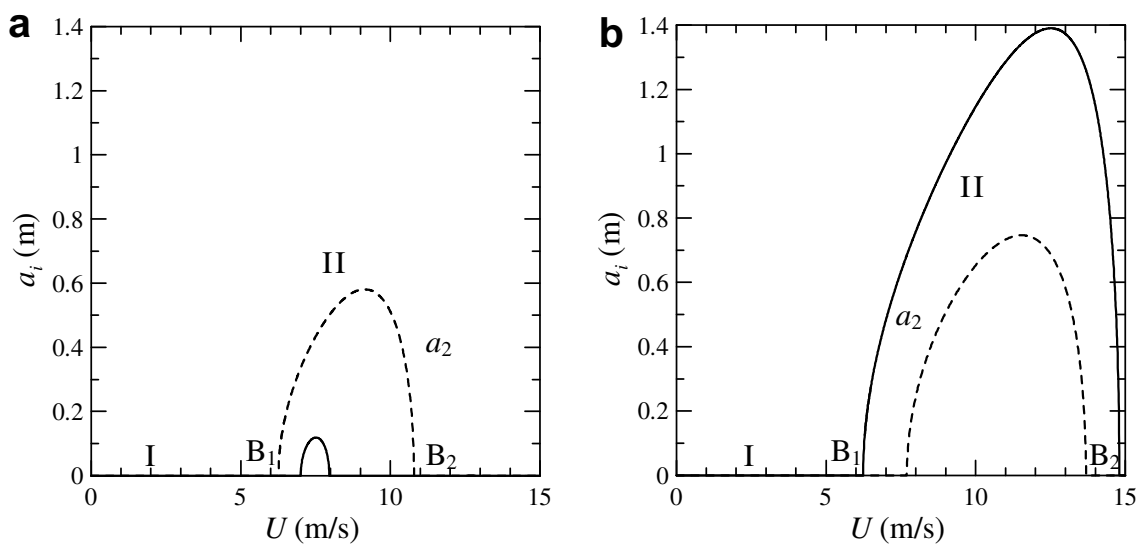

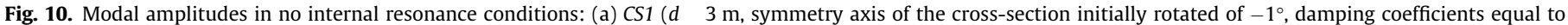

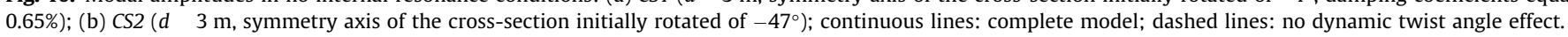

partially taken into account. This occurs because of changes in the aerodynamic coefficients, which do not remain constant (at the most dangerous values, as in [3]) when the mean velocity (i.e. the static rotation $\varphi$ ) increases. Therefore, in internal resonant con ditions, branch III can only be obtained if the detuning is substan tially small (examples seem very sensitive to negative detuning) and the cross section is suitably rotated in no wind conditions (which helps the occurrence of oscillations of sufficiently high in plane amplitudes).

Concerning the non resonant case, the two systems already studied (see Fig. 7 and Luongo et al. [10]) in the linear range ( $d \quad 3 \mathrm{~m}$ ) are reconsidered in Fig. 10. For both these examples the sole branch II exists, at least in the range of velocities considered here. Moreover, differences induced by the dynamic twist are very remarkable. The possible presence of a branch III has been checked through methods discussed in Section 4.1. For both the cross sec tions studied, a branch III, independent from the mono modal branch II, has been discovered at high mean velocities only (of about $20 \mathrm{~m} / \mathrm{s}$ ). Moreover, the presence of the twist angle modifies also the cable frequencies and shifts the resonance; so, an initial non reso nant problem (i.e. cable with a small but finite sag) can become $1: 1$ resonant when velocities suitably increases. For the systems investigated here, this phenomenon occurs at a mean wind velocity of about $23 \mathrm{~m} / \mathrm{s}$ (Fig. 11). Such a kind of branch III, however, cannot be studied with the proposed methods, but it requires specific treat ments which go beyond the objectives of the present paper.

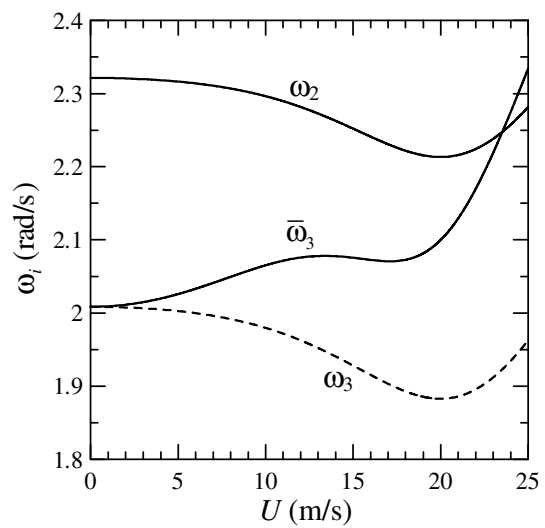

Fig. 11. In-plane and out-of-plane cable frequencies (CS2, $d \quad 3 \mathrm{~m}$, symmetry axis of the cross-section initially rotated of $-47^{\circ}$ ).

\section{Conclusions}

In this paper, a nonlinear model of cable beam has been formu lated, accounting for both torsion and bending. After a consistent analysis of the order of magnitude of the terms (mainly based on the hypotheses of large slenderness ratio, small initial curvature and quasi steady stretching and twisting), a set of reduced inte gro differential equations of motion has been obtained, which 
captures the essential dynamics of the cable. As a main result, it has been proved that, while the bending stiffness does not significantly affect the translational dynamics, it, in contrast, contributes to twist ing, since bending and torcent moments are coupled by equilibrium.

The model is able to describe the dependence of the aerody namic forces on the actual, time dependent, cross section attitude, which consists of a large static contribution produced by the stea dy wind forces, and a small but finite dynamic contribution, de scribed by the centerline velocity and the twist rotation. Therefore, linear velocity dependent and circulatory (twist depen dent) forces act on the body, together with nonlinear forces, of qua dratic and cubic type, depending on any combinations of structural velocities and twist.

A simplified two degree of freedom system, drawn by the con tinuous one by a Galerkin procedure, has been used to investigate the critical and post critical aeroelastic behavior of the cable. By applying the Multiple Scale perturbation method, an equivalent amplitude phase set of equations has been drawn, both for $2: 1$ internally resonant systems, i.e. for cables close to the so called first cross over point, and for not internally resonant systems, i.e. for cables with smaller sag. The parametric study carried out lead to the following conclusions.

1. The static twist angle remarkably modifies the natural frequen cies of the cable, through alteration of the internal prestress. In particular, cables that are not resonant in absence of wind can become 1:1 resonant under static wind forces (resonance shift phenomenon). Moreover, the static rotation changes the section aerodynamic properties and, consequently, the critical wind velocity value. Such modifications are more significant when a section initially non symmetric with respect to the wind direc tion is considered.

2. The influence of the dynamic twist on the critical velocity is appreciable when galloping modes are of symmetric type; it can become remarkable for non symmetric cross sections in no wind conditions.

3. The post critical scenario of $2: 1$ internally resonant case is not qualitatively modified by twisting (except for particular cases where the dynamic twist is responsible for bifurcations). Limit cycles of (a) mono modal in plane oscillations and (b) bi modal, in plane and out of plane coupled oscillations are found, simi lar to that ones exhibited by the perfectly flexible model. How ever, significant quantitative changes are noticed.

4. Even more pronounced differences between the two models exist for non resonant cables. Here, only mono modal solutions were found at low wind velocities. Bi modal oscillations also exist at higher velocity where, however, the resonance shift invalidates the non resonant solution.

\section{Acknowledgements}

This work has been partially supported by a PRIN grant (http://www.disg.uniromal.it/fendis) and by the INTAS Pro ject no. 0610000139019 (www.intas.be).

\section{Appendix A. Quasi-steady twist}

Since the torsional frequencies of a single cable turn out to be much higher than the transversal ones, the inertia couple $\mathscr{J}_{1} \vartheta$ and the damping couple $c_{\vartheta} \dot{\vartheta}$ are neglected in Eq. (6) $)_{\mathrm{d}}$. To solve this equation with the boundary conditions (7) in the unknown $\vartheta$, $v$ and $w$ are first scaled by a perturbation parameter $\epsilon \ll 1$, then $\vartheta$ is expanded in $\epsilon$ series:

$v \rightarrow \epsilon v, \quad w \rightarrow \epsilon W, \quad \vartheta \quad \epsilon \vartheta_{1}+\epsilon^{2} \vartheta_{2}+\cdots$
The following perturbation equations are obtained:

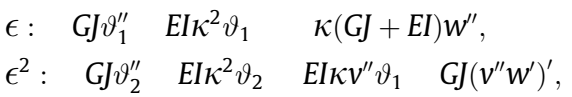

where $c_{1} \quad 0$ has been taken for simplicity. The relevant boundary conditions read:

$$
\begin{array}{lll}
\epsilon: & G J\left(\vartheta_{1}^{\prime}+\kappa w^{\prime}\right) & 0, \\
\epsilon^{2}: & G J\left(\vartheta_{2}^{\prime}+v^{\prime \prime} w^{\prime}\right) & 0 .
\end{array}
$$

By solving in chain Eq. (33), it follows:

$$
\begin{aligned}
\vartheta_{1}(s, t) \quad & \frac{G J+E I}{\sqrt{ } G J E I} \int_{0}^{s} w^{\prime \prime}(\xi, t) \sinh \left[k\left(\begin{array}{ll}
s & \xi
\end{array}\right)\right] \mathrm{d} \xi \\
+ & A_{1} \cosh k s+B_{1} \sinh k s, \\
\vartheta_{2}(s, t) \quad & \sqrt{\frac{E I}{G J} \int_{0}^{s} v^{\prime \prime}(\zeta, t) \vartheta_{1}(\zeta, t) \sinh \left[\begin{array}{ll}
s & \zeta
\end{array}\right] \mathrm{d} \zeta} \\
& \left.\frac{1}{k} \int_{0}^{s}\left(v^{\prime \prime}(\xi, t) w^{\prime}(\xi, t)\right)^{\prime} \sinh \left[\begin{array}{ll}
s & \xi
\end{array}\right)\right] \mathrm{d} \xi \\
+ & A_{2} \cosh k s+B_{2} \sinh k s,
\end{aligned}
$$

where $k: \quad \bar{\kappa} \sqrt{ } E I / G J$ has been set and where the arbitrary constants $A_{1}, B_{1}, A_{2}, B_{2}$ are determined by Eq. (34). By substituting Eq. (35) in $(32)_{c}$ and reabsorbing the perturbation parameter, Eq. (11) is drawn.

\section{Appendix B. Static wind forces and aerodynamic coefficients}

The static wind force components $\bar{b}_{a_{i}}$ and the coefficient c's expressing the dynamic wind force components $\left(b_{a_{i}} \quad \bar{b}_{a_{i}}\right)$, all appearing in Eq. (15), are listed below $\left(\begin{array}{ll}k & 0.5 \rho_{a} r\end{array}\right)$ :

$$
\begin{aligned}
& b_{a_{2}} k U^{2}\left(c_{1} \cos \varphi+c_{\mathrm{d}} \sin \varphi\right), \quad c_{21} \quad k U^{2}\left(c_{\mathrm{d}}^{\prime} \sin \varphi+c_{1}^{\prime} \cos \varphi\right), \\
& c_{22} \quad k U\left(c_{\mathrm{d}} \quad c_{1} \cos \varphi \sin \varphi+c_{\mathrm{d}} \sin ^{2} \varphi\right. \\
& \left.c_{\mathrm{d}}^{\prime} \cos \varphi \sin \varphi+c_{1}^{\prime} \cos ^{2} \varphi\right) \text {, } \\
& c_{23} \quad k U\left(c_{1}+c_{1} \cos ^{2} \varphi \quad c_{\mathrm{d}} \cos \varphi \sin \varphi \quad c_{\mathrm{d}}^{\prime} \sin ^{2} \varphi+c_{1}^{\prime} \cos \varphi \sin \varphi\right), \\
& c_{211} \quad 0.5 k U^{2}\left(c_{\mathrm{d}}^{\prime \prime} \sin \varphi \quad c_{1}^{\prime \prime} \cos \varphi\right) \text {, } \\
& c_{212} k U\left\{c_{\mathrm{d}}^{\prime}\left(1+\sin ^{2} \varphi\right)+\cos \varphi\left[\left(c_{1}^{\prime}+c_{\mathrm{d}}^{\prime \prime}\right) \sin \varphi+c_{1}^{\prime \prime} \cos \varphi\right]\right\}, \\
& c_{213} \quad k U\left[c_{\mathrm{d}}^{\prime} \cos \varphi \sin \varphi+c_{1}^{\prime}\left(1+\cos ^{2} \varphi\right)\right. \\
& \left.+\sin \varphi\left(c_{\mathrm{d}}^{\prime \prime} \sin \varphi+c_{1}^{\prime \prime} \cos \varphi\right)\right] \text {, } \\
& c_{222} \quad 0.125 k\left[4 c_{1} \cos ^{3} \varphi \quad c_{\mathrm{d}}(9 \sin \varphi+\sin 3 \varphi)+8 c_{\mathrm{d}}^{\prime} \cos \varphi\right. \\
& \left.4 c_{\mathrm{d}}^{\prime \prime} \cos ^{2} \varphi \sin \varphi+4 c_{1}^{\prime \prime} \cos ^{3} \varphi\right] \text {, } \\
& c_{223} \quad k\left\{c_{\mathrm{d}} \cos ^{3} \varphi \quad c_{1} \sin ^{3} \varphi+c_{\mathrm{d}}^{\prime} \sin \varphi\right. \\
& \left.+\cos \varphi\left[c_{1}^{\prime}+\sin \varphi\left(c_{\mathrm{d}}^{\prime \prime} \sin \varphi+c_{1}^{\prime \prime} \cos \varphi\right)\right]\right\}, \\
& c_{233} \quad 0.125 k\left(9 c_{1} \cos \varphi \quad c_{1} \cos 3 \varphi \quad 4 c_{\mathrm{d}} \sin ^{3} \varphi+8 c_{1}^{\prime} \sin \varphi\right. \\
& \left.4 c_{\mathrm{d}}^{\prime \prime} \sin ^{3} \varphi+4 c_{1}^{\prime \prime} \cos \varphi \sin ^{2} \varphi\right) \text {, } \\
& c_{2111} k U^{2}\left(c_{\mathrm{d}}^{\prime \prime \prime} \sin \varphi+c_{1}^{\prime \prime \prime} \cos \varphi\right) / 6, \\
& c_{2112} \quad 0.25 k U\left\{c_{d}^{\prime \prime}(3+\cos 2 \varphi)\right. \\
& \left.+2 \cos \varphi\left[\left(c_{1}^{\prime \prime}+c_{d}^{\prime \prime \prime}\right) \sin \varphi \quad c_{1}^{\prime \prime \prime} \cos \varphi\right]\right\}, \\
& c_{2113} \quad 0.25 k U\left[c_{1}^{\prime \prime}(3+\cos 2 \varphi) \quad 2 c_{d}^{\prime \prime \prime} \sin ^{2} \varphi+\sin 2 \varphi\left(c_{d}^{\prime \prime}+c_{1}^{\prime \prime \prime}\right)\right] \text {, } \\
& c_{2122} \quad 0.125 k\left\{c_{\mathrm{d}}^{\prime}(9 \sin \varphi+\sin 3 \varphi)\right. \\
& \left.4 \cos \varphi\left[2 c_{\mathrm{d}}^{\prime \prime}+\cos \varphi\left(c_{\mathrm{d}}^{\prime \prime \prime} \sin \varphi+\left(c_{1}^{\prime}+c_{1}^{\prime \prime \prime}\right) \cos \varphi\right)\right]\right\} \text {, } \\
& c_{2123} \quad k\left\{c_{\mathrm{d}}^{\prime} \cos ^{3} \varphi+c_{1}^{\prime \prime} \cos \varphi\right. \\
& \left.+\sin \varphi\left[c_{\mathrm{d}}^{\prime \prime} \quad \sin \varphi\left(c_{1}^{\prime} \sin \varphi+c_{\mathrm{d}}^{\prime \prime \prime} \cos \varphi\right)+c_{1}^{\prime \prime \prime} \cos ^{2} \varphi\right]\right\}, \\
& 0.125 k\left\{c_{1}^{\prime}(9 \cos \varphi+\cos 3 \varphi)\right. \\
& \left.+4 \sin \varphi\left[2 c_{1}^{\prime \prime}+\sin \varphi\left(\left(c_{\mathrm{d}}^{\prime}+c_{\mathrm{d}}^{\prime \prime \prime}\right) \sin \varphi \quad c_{1}^{\prime \prime \prime} \cos \varphi\right)\right]\right\},
\end{aligned}
$$


$c_{2222} \quad\left\{k \cos ^{3} \varphi\left[\begin{array}{ll}\left(c_{\mathrm{d}}^{\prime}\right. & \left.3\left(c_{1}+c_{1}^{\prime \prime}\right)+c_{\mathrm{d}}^{\prime \prime \prime}\right) \sin \varphi\end{array}\right.\right.$ $\left.\left.+\left(c_{1}^{\prime}+3\left(c_{\mathrm{d}}+c_{\mathrm{d}}^{\prime \prime}\right)+c_{1}^{\prime \prime \prime}\right) \cos \varphi\right]\right\} /(6 U)$,

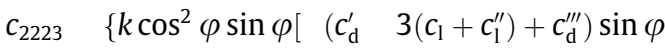
$\left.\left.+\left(c_{1}^{\prime}+3\left(c_{\mathrm{d}}+c_{\mathrm{d}}^{\prime \prime}\right)+c_{1}^{\prime \prime \prime}\right) \cos \varphi\right]\right\} /(2 U)$,

$c_{2233} \quad\left\{k \cos \varphi \sin ^{2} \varphi\left[\begin{array}{ll}\left(c_{\mathrm{d}}^{\prime}\right. & \left.3\left(c_{1}+c_{1}^{\prime \prime}\right)+c_{\mathrm{d}}^{\prime \prime \prime}\right) \sin \varphi\end{array}\right.\right.$ $\left.\left.+\left(c_{1}^{\prime}+3\left(c_{\mathrm{d}}+c_{\mathrm{d}}^{\prime \prime}\right)+c_{1}^{\prime \prime \prime}\right) \cos \varphi\right]\right\} /(2 U)$,

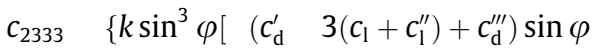
$\left.\left.+\left(c_{1}^{\prime}+3\left(c_{\mathrm{d}}+c_{\mathrm{d}}^{\prime \prime}\right)+c_{1}^{\prime \prime \prime}\right) \cos \varphi\right]\right\} /(6 U)$,

$b_{a_{3}} \quad k U^{2}\left(c_{\mathrm{d}} \cos \varphi+c_{1} \sin \varphi\right), \quad c_{31} \quad k U^{2}\left(c_{\mathrm{d}}^{\prime} \cos \varphi+c_{1}^{\prime} \sin \varphi\right)$,

$c_{32} \quad k U\left(c_{1}+c_{\mathrm{d}} \cos \varphi \sin \varphi+c_{1} \sin ^{2} \varphi\right.$ $\left.c_{\mathrm{d}}^{\prime} \cos ^{2} \varphi \quad c_{1}^{\prime} \cos \varphi \sin \varphi\right)$,

$c_{33} \quad k U\left(c_{\mathrm{d}}+c_{\mathrm{d}} \cos ^{2} \varphi+c_{1} \cos \varphi \sin \varphi\right.$

$\left.+c_{\mathrm{d}}^{\prime} \cos \varphi \sin \varphi+c_{1}^{\prime} \sin ^{2} \varphi\right)$,

$c_{311} \quad 0.5 k U^{2}\left(c_{\mathrm{d}}^{\prime \prime} \cos \varphi+c_{1}^{\prime \prime} \sin \varphi\right)$,

$c_{312} \quad k U\left\{c_{\mathrm{d}}^{\prime} \cos \varphi \sin \varphi+\left(1+\sin ^{2} \varphi\right) c_{1}^{\prime}\right.$ $\left.\cos \varphi\left[c_{\mathrm{d}}^{\prime \prime} \cos \varphi+c_{1}^{\prime \prime} \sin \varphi\right]\right\}$,

$c_{313} k U\left(c_{\mathrm{d}}^{\prime}\left(1+\cos ^{2} \varphi\right)+\sin \varphi\left[\left(c_{1}^{\prime}+c_{\mathrm{d}}^{\prime \prime}\right) \cos \varphi+c_{1}^{\prime \prime} \sin \varphi\right]\right)$,

$c_{322} \quad 0.125 k\left[4 c_{\mathrm{d}} \cos ^{3} \varphi+c_{1}(9 \sin \varphi+\sin 3 \varphi) \quad 8 c_{1}^{\prime} \cos \varphi\right.$

$\left.+4 c_{\mathrm{d}}^{\prime \prime} \cos ^{3} \varphi+4 c_{1}^{\prime \prime} \cos ^{2} \varphi \sin \varphi\right]$,

$c_{323} k\left\{c_{1} \cos ^{3} \varphi+c_{\mathrm{d}} \sin ^{3} \varphi+c_{1}^{\prime} \sin \varphi\right.$ $\left.\cos \varphi\left[c_{\mathrm{d}}^{\prime}+\sin \varphi\left(c_{\mathrm{d}}^{\prime \prime} \cos \varphi+c_{1}^{\prime \prime} \sin \varphi\right)\right]\right\}$

$c_{333}$

$0.125 k\left(9 c_{\mathrm{d}} \cos \varphi \quad c_{\mathrm{d}} \cos 3 \varphi+4 c_{1} \sin ^{3} \varphi+8 c_{\mathrm{d}}^{\prime} \sin \varphi\right.$

$\left.+4 c_{\mathrm{d}}^{\prime \prime} \sin ^{2} \varphi \cos \varphi+4 c_{1}^{\prime \prime} \sin ^{3} \varphi\right)$,

$c_{3111} \quad k U^{2}\left(c_{\mathrm{d}}^{\prime \prime \prime} \cos \varphi+c_{1}^{\prime \prime \prime} \sin \varphi\right) / 6$,

$c_{3112} \quad 0.25 k U\left[c_{1}^{\prime \prime}(3+\cos 2 \varphi)+2 c_{d}^{\prime \prime \prime} \cos ^{2} \varphi+\left(c_{d}^{\prime \prime}+c_{1}^{\prime \prime \prime}\right) \sin 2 \varphi\right]$,

$c_{3113} \quad 0.25 k U\left\{c_{\mathrm{d}}^{\prime \prime}(3+\cos 2 \varphi)+2 \sin \varphi\left[\left(c_{1}^{\prime \prime}+c_{\mathrm{d}}^{\prime \prime \prime}\right) \cos \varphi\right.\right.$

$\left.\left.+c_{1}^{\prime \prime \prime} \sin \varphi\right]\right\}$,

$c_{3122} \quad 0.125 k\left\{c_{1}^{\prime}(9 \sin \varphi+\sin 3 \varphi)+4 \cos \varphi\left[2 c_{1}^{\prime \prime}\right.\right.$

$\left.\left.+\cos \varphi\left(c_{1}^{\prime \prime \prime} \sin \varphi+\left(c_{\mathrm{d}}^{\prime}+c_{\mathrm{d}}^{\prime \prime \prime}\right) \cos \varphi\right)\right]\right\}$,

$c_{3123} \quad k\left\{c_{\mathrm{d}}^{\prime} \sin ^{3} \varphi+c_{1}^{\prime} \cos ^{3} \varphi+c_{1}^{\prime \prime} \sin \varphi\right.$ $\left.\cos \varphi\left[c_{\mathrm{d}}^{\prime \prime}+c_{\mathrm{d}}^{\prime \prime \prime} \cos \varphi \sin \varphi+c_{1}^{\prime \prime \prime} \sin ^{2} \varphi\right]\right\}$,

$c_{3133} \quad 0.125 k\left\{c_{\mathrm{d}}^{\prime}(9 \cos \varphi+\cos 3 \varphi)\right.$ $\left.+4 \sin \varphi\left[2 c_{\mathrm{d}}^{\prime \prime}+\sin \varphi\left(\left(c_{1}^{\prime}+c_{1}^{\prime \prime \prime}\right) \sin \varphi+c_{\mathrm{d}}^{\prime \prime \prime} \cos \varphi\right)\right]\right\}$,

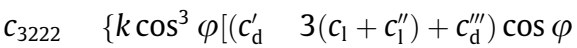
$\left.\left.+\left(c_{1}^{\prime}+3\left(c_{\mathrm{d}}+c_{\mathrm{d}}^{\prime \prime}\right)+c_{1}^{\prime \prime \prime}\right) \sin \varphi\right]\right\} /(6 U)$,

$c_{3223} \quad\left\{k \cos ^{2} \varphi \sin \varphi\left[\left(c_{\mathrm{d}}^{\prime} \quad 3\left(c_{1}+c_{1}^{\prime \prime}\right)+c_{\mathrm{d}}^{\prime \prime \prime}\right) \cos \varphi\right.\right.$ $\left.\left.+\left(c_{1}^{\prime}+3\left(c_{\mathrm{d}}+c_{\mathrm{d}}^{\prime \prime}\right)+c_{1}^{\prime \prime \prime}\right) \sin \varphi\right]\right\} /(2 U)$,

$c_{3233}\left\{k \cos \varphi \sin ^{2} \varphi\left[\left(c_{\mathrm{d}}^{\prime} 3\left(c_{1}+c_{1}^{\prime \prime}\right)+c_{\mathrm{d}}^{\prime \prime \prime}\right) \cos \varphi\right.\right.$ $\left.\left.+\left(c_{1}^{\prime}+3\left(c_{\mathrm{d}}+c_{\mathrm{d}}^{\prime \prime}\right)+c_{1}^{\prime \prime \prime}\right) \sin \varphi\right]\right\} /(2 U)$,

$c_{3333} \quad\left\{k \sin ^{3} \varphi\left[\left(c_{\mathrm{d}}^{\prime} \quad 3\left(c_{1}+c_{1}^{\prime \prime}\right)+c_{\mathrm{d}}^{\prime \prime \prime}\right) \cos \varphi\right.\right.$

$\left.\left.+\left(c_{1}^{\prime}+3\left(c_{\mathrm{d}}+c_{\mathrm{d}}^{\prime \prime}\right)+c_{1}^{\prime \prime \prime}\right) \sin \varphi\right]\right\} /(6 U)$.

\section{Appendix C. Coefficients of the discrete model}

The aerodynamic and mechanical coefficients of the discrete model (17) are

$\omega_{2}^{2} \quad E A \frac{\kappa^{2}}{m_{2} \ell}\left[\int_{0}^{\ell} \phi_{v} \mathrm{~d} s\right]^{2}+\frac{\bar{T}}{m_{2}} \int_{0}^{\ell} \phi_{v}^{\prime \prime} \phi_{v} \mathrm{~d} s, \quad h_{23} \quad \frac{c_{21}}{m_{2}} \int_{0}^{\ell} \phi_{v} \phi_{\vartheta} \mathrm{d} s$,

$c_{a 22} \frac{c_{22}}{m_{2}} \int_{0}^{\ell} \phi_{v}^{2} \mathrm{~d} s, \quad c_{a 23} \quad \frac{c_{23}}{m_{2}} \int_{0}^{\ell} \phi_{v} \phi_{w} \mathrm{~d} s$, $h_{1} \frac{E A \kappa}{2 m_{2} \ell} \int_{0}^{\ell} \phi_{v} \mathrm{~d} s \cdot \int_{0}^{\ell} \phi_{v}^{\prime 2} \mathrm{~d} s \frac{E A \kappa}{m_{2} \ell} \int_{0}^{\ell} \phi_{v}^{\prime \prime} \phi_{v} \mathrm{~d} s \cdot \int_{0}^{\ell} \phi_{v} \mathrm{~d} s$,

$h_{2} \frac{E A \kappa}{2 m_{2} \ell} \int_{0}^{\ell} \phi_{v} \mathrm{~d} s \cdot \int_{0}^{\ell} \phi_{w}^{\prime 2} \mathrm{~d} s+\frac{c_{211}}{m_{2}} \int_{0}^{\ell} \phi_{v} \phi_{\vartheta}^{2} \mathrm{~d} s$,

$h_{3} \frac{E A}{2 m_{2} \ell} \int_{0}^{\ell} \phi_{v}^{\prime \prime} \phi_{v} \mathrm{~d} s \cdot \int_{0}^{\ell} \phi_{w}^{\prime 2} \mathrm{~d} s$,

$h_{4} \quad \frac{E A}{2 m_{2} \ell} \int_{0}^{\ell} \phi_{v}^{\prime \prime} \phi_{v} \mathrm{~d} s \cdot \int_{0}^{\ell} \phi_{v}^{\prime 2} \mathrm{~d} s$,

$h_{5} \frac{c_{2111}}{m_{2}} \int_{0}^{\ell} \phi_{v} \phi_{\vartheta}^{3} \mathrm{~d} s, \quad d_{1} \frac{c_{212}}{m_{2}} \int_{0}^{\ell} \phi_{v}^{2} \phi_{\vartheta} \mathrm{d} s$,

$d_{2} \frac{c_{213}}{m_{2}} \int_{0}^{\ell} \phi_{v} \phi_{w} \phi_{\vartheta} \mathrm{d} s$,

$d_{3} \frac{c_{222}}{m_{2}} \int_{0}^{\ell} \phi_{v}^{3} \mathrm{~d} s, \quad d_{4} \quad \frac{c_{223}}{m_{2}} \int_{0}^{\ell} \phi_{v}^{2} \phi_{w} \mathrm{~d} s, \quad d_{5} \quad \frac{c_{233}}{m_{2}} \int_{0}^{\ell} \phi_{v} \phi_{w}^{2} \mathrm{~d} s$,

$d_{6} \frac{c_{2112}}{m_{2}} \int_{0}^{\ell} \phi_{v}^{2} \phi_{\vartheta}^{2} \mathrm{~d} s, \quad d_{7} \frac{c_{2113}}{m_{2}} \int_{0}^{\ell} \phi_{v} \phi_{w} \phi_{\vartheta}^{2} \mathrm{~d} s$,

$d_{8} \frac{c_{2122}}{m_{2}} \int_{0}^{\ell} \phi_{v}^{3} \phi_{\vartheta} \mathrm{d} s$,

$d_{9} \frac{c_{2123}}{m_{2}} \int_{0}^{\ell} \phi_{v}^{2} \phi_{w} \phi_{\vartheta} \mathrm{d} s, \quad d_{10} \quad \frac{c_{2133}}{m_{2}} \int_{0}^{\ell} \phi_{v} \phi_{w}^{2} \phi_{\vartheta} \mathrm{d} s$,

$d_{11} \frac{c_{2222}}{m_{2}} \int_{0}^{\ell} \phi_{v}^{4} \mathrm{ds}$,

$d_{12} \frac{c_{2223}}{m_{2}} \int_{0}^{\ell} \phi_{v}^{3} \phi_{w} \mathrm{~d} s, \quad d_{13} \frac{c_{2233}}{m_{2}} \int_{0}^{\ell} \phi_{v}^{2} \phi_{w}^{2} \mathrm{~d} s$,

$d_{14} \frac{c_{2333}}{m_{2}} \int_{0}^{\ell} \phi_{v} \phi_{w}^{3} \mathrm{~d} s$,

$m_{2} \quad m \int_{0}^{\ell} \phi_{v}^{2} \mathrm{~d} s$,

$\omega_{3}^{2} \frac{\bar{T}}{m_{3}} \int_{0}^{\ell} \phi_{w}^{\prime \prime} \phi_{w} \mathrm{~d} s, \quad h_{33} \quad \frac{c_{31}}{m_{3}} \int_{0}^{\ell} \phi_{w} \phi_{\vartheta} \mathrm{d} s$,

$c_{a 32} \frac{c_{32}}{m_{3}} \int_{0}^{\ell} \phi_{v} \phi_{w} \mathrm{~d} s, \quad c_{a 33} \frac{c_{33}}{m_{3}} \int_{0}^{\ell} \phi_{w}^{2} \mathrm{~d} s$,

$h_{6} \quad \frac{E A \kappa}{m_{3} \ell} \int_{0}^{\ell} \phi_{w}^{\prime \prime} \phi_{w} \mathrm{~d} s \cdot \int_{0}^{\ell} \phi_{v} \mathrm{~d} s \quad \frac{E I \kappa}{m_{3}} \int_{0}^{\ell} \phi_{v}^{\prime \prime} \phi_{v}^{2} \mathrm{~d} s$

$+\frac{G J}{m_{3}}\left[\int_{0}^{\ell} \phi_{v}^{\prime \prime \prime} \phi_{w}^{\prime} \phi_{\vartheta} \mathrm{d} s+\int_{0}^{\ell} \phi_{v}^{\prime \prime} \phi_{w}^{\prime \prime} \phi_{\vartheta} \mathrm{d} s\right]$,

$h_{7} \frac{c_{311}}{m_{3}} \int_{0}^{\ell} \phi_{w} \phi_{\vartheta}^{2} \mathrm{~d} s, \quad h_{8} \quad \frac{E A}{2 m_{3} \ell} \int_{0}^{\ell} \phi_{w}^{\prime \prime} \phi_{w} \mathrm{~d} s \cdot \int_{0}^{\ell} \phi_{v}^{\prime 2} \mathrm{~d} s$,

$h_{9} \quad \frac{E A}{2 m_{3} \ell} \int_{0}^{\ell} \phi_{w}^{\prime \prime} \phi_{w} \mathrm{~d} s \cdot \int_{0}^{\ell} \phi_{w}^{\prime 2} \mathrm{~d} s+c_{3111} \int_{0}^{\ell} \phi_{w} \phi_{\vartheta}^{3} \mathrm{~d} s$,

$d_{15} \frac{c_{312}}{m_{3}} \int_{0}^{\ell} \phi_{v} \phi_{w} \phi_{\vartheta} \mathrm{d} s, \quad d_{16} \quad \frac{c_{313}}{m_{3}} \int_{0}^{\ell} \phi_{w}^{2} \phi_{\vartheta} \mathrm{d} s$,

$d_{17} \frac{c_{322}}{m_{3}} \int_{0}^{\ell} \phi_{v}^{2} \phi_{w} \mathrm{ds}$,

$d_{18} \frac{c_{323}}{m_{3}} \int_{0}^{\ell} \phi_{v} \phi_{w}^{2} \mathrm{~d} s, \quad d_{19} \quad \frac{c_{333}}{m_{3}} \int_{0}^{\ell} \phi_{w}^{3} \mathrm{~d} s$,

$d_{20} \frac{c_{3112}}{m_{3}} \int_{0}^{\ell} \phi_{v} \phi_{w} \phi_{\vartheta}^{2} \mathrm{~d} s$,

$d_{21} \frac{c_{3113}}{m_{3}} \int_{0}^{\ell} \phi_{w}^{2} \phi_{\vartheta}^{2} \mathrm{~d} s, \quad d_{22} \quad \frac{c_{3122}}{m_{3}} \int_{0}^{\ell} \phi_{v}^{2} \phi_{w} \phi_{\vartheta} \mathrm{d} s$,

$d_{23} \frac{c_{3123}}{m_{3}} \int_{0}^{\ell} \phi_{v} \phi_{w}^{2} \phi_{\vartheta} \mathrm{d} s$,

$d_{24} \frac{c_{3133}}{m_{3}} \int_{0}^{\ell} \phi_{w}^{3} \phi_{\vartheta} \mathrm{d} s, \quad d_{25} \quad \frac{c_{3222}}{m_{3}} \int_{0}^{\ell} \phi_{v}^{3} \phi_{w} \mathrm{~d} s$,

$d_{26} \quad \frac{c_{3223}}{m_{3}} \int_{0}^{\ell} \phi_{v}^{2} \phi_{w}^{2} \mathrm{~d} s$,

$d_{27} \quad \frac{c_{3233}}{m_{3}} \int_{0}^{\ell} \phi_{v} \phi_{w}^{3} \mathrm{~d} s, \quad d_{28} \quad \frac{c_{3333}}{m_{3}} \int_{0}^{\ell} \phi_{w}^{4} \mathrm{~d} s$,

$m_{3} \quad m \int_{0}^{\ell} \phi_{w}^{2} \mathrm{~d} s$. 


\section{Appendix D. Eigenvectors of the matrix of the generating problem}

The eigenvalue problem for the matrix of the generating problem (19)

$$
\left[\begin{array}{cc}
\omega_{2}^{2} & h_{23} \\
0 & \bar{\omega}_{3}^{3}
\end{array}\right]\left(\begin{array}{l}
u_{21} \\
u_{31}
\end{array}\right)=\left(\begin{array}{l}
0 \\
0
\end{array}\right)
$$

leads to the eigenvalues $\pm i \omega_{2}, \pm i \bar{\omega}_{3}$ and the corresponding right eigenvectors $\mathbf{u}$ :

$$
\mathbf{u}_{2}=\left(\begin{array}{l}
1 \\
0
\end{array}\right), \quad \mathbf{u}_{3}=\left(\begin{array}{c}
-\frac{h_{23}}{\omega_{2}^{2}-\bar{\omega}_{3}^{2}} \\
1
\end{array}\right) .
$$

The eigenvalue problem for the transpose of the matrix of the generating problem (19) leads to the same eigenvalues but to different eigenvectors, called left eigenvectors v:

$\mathbf{v}_{2}=\left(\begin{array}{c}1 \\ \frac{h_{23}}{\omega_{2}^{2}-\bar{\omega}_{3}^{2}}\end{array}\right), \quad \mathbf{v}_{3}=\left(\begin{array}{l}0 \\ 1\end{array}\right)$.

\section{Appendix E. AME's linear coefficients}

The linear coefficients p's of the AME are:

$$
\begin{aligned}
p_{10}= & -\frac{c_{a 22}}{2}-\zeta_{2} \omega_{2}-\frac{c_{a 32} h_{23}}{2\left(\omega_{2}^{2}-\bar{\omega}_{3}^{2}\right)}, \\
p_{50}= & -\frac{c_{a 33}}{2}-\zeta_{3} \omega_{3}+\frac{c_{a 32} h_{23}}{2\left(\omega_{2}^{2}-\bar{\omega}_{3}^{2}\right)}, \\
p_{12}= & -\frac{c_{a 23} \Lambda_{23}}{2}+\frac{h_{23} \Lambda_{23}\left(c_{a 22}-c_{a 33}+2 \zeta_{2} \omega_{2}-2 \zeta_{3} \omega_{3}\right)}{2\left(\omega_{2}^{2}-\bar{\omega}_{3}^{2}\right)} \\
& +\frac{h_{23}^{2} c_{a 32} \Lambda_{23}}{2\left(\omega_{2}^{2}-\bar{\omega}_{3}^{2}\right)^{2}} \\
& -\frac{c_{a 22}^{2}+\left(2 \zeta_{2} \omega_{2}\right)^{2}}{8 \omega_{2}}-\frac{c_{a 22}\left(2 \zeta_{2} \omega_{2}\right)}{4 \omega_{2}}-\frac{c_{a 32} h_{23}\left(c_{a 22}+2 \zeta_{2} \omega_{2}\right)}{4 \omega_{2}\left(\omega_{2}^{2}-\bar{\omega}_{3}^{2}\right)} \\
& -\frac{c_{a 32}^{2} h_{23}^{2}}{8 \omega_{2}\left(\omega_{2}^{2}-\bar{\omega}_{3}^{2}\right)^{2}}, \\
p_{52}= & -\frac{c_{a 32} \Lambda_{14}}{2}-\frac{c_{a 33}^{2}+\left(2 \zeta_{3} \omega_{3}\right)^{2}}{8 \omega_{3}}-\frac{c_{a 33}\left(2 \zeta_{3} \omega_{3}\right)}{4 \omega_{3}} \\
& +\frac{c_{a 32} h_{23}\left(c_{a 33}+2 \zeta_{3} \omega_{3}\right)}{4 \omega_{3}\left(\omega_{2}^{2}-\bar{\omega}_{3}^{2}\right)}-\frac{c_{a 32}^{2} h_{23}^{2}}{8 \omega_{3}\left(\omega_{2}^{2}-\bar{\omega}_{3}^{2}\right)^{2}}, \\
\Lambda_{23}= & \frac{c_{a 32} \omega_{2}}{\left(\omega_{2}^{2}-\bar{\omega}_{3}^{2}\right)}, \\
\Lambda_{14}= & -\frac{c_{a 23} \omega_{3}}{\left(\omega_{2}^{2}-\bar{\omega}_{3}^{2}\right)}+\frac{h_{23} \omega_{3}\left(c_{a 22}-c_{a 33}+2 \zeta_{2} \omega_{2}-2 \zeta_{3} \omega_{3}\right)}{\left(\omega_{2}^{2}-\bar{\omega}_{3}^{2}\right)^{2}} \\
& +\frac{c_{a 32} h_{23}^{2} \omega_{3}}{\left(\omega_{2}^{2}-\bar{\omega}_{3}^{2}\right)^{3}} .
\end{aligned}
$$

\section{References}

[1] Novak M. Aeroelastic galloping of prismatic bodies. J Eng Mech, ASCE 1969;95(EM1):115-41.

[2] Blevins RD. Flow-induced vibration. 2nd ed. Malabar (FL): Krieger Publishing Company; 2001.

[3] Luongo A, Piccardo G. Non-linear galloping of sagged cables in 1:2 internal resonance. J Sound Vib 1998;214(5):915-40.

[4] Luongo A, Rega G, Vestroni F. Planar non-linear free vibrations of an elastic cable. Int J Non-Linear Mech 1984;19(1):39-52.

[5] Lee CL, Perkins NC. Nonlinear oscillations of suspended cables containing a two-to-one internal resonance. Nonlinear Dyn 1992;3:465-90.

[6] Luongo A, Piccardo G. On the influence of the torsional stiffness on non-linear galloping of suspended cables. In: Proceedings of the second ENOC, Prague, Czech Republic, vol. 1; 1996. p. 273-6.

[7] Yu P, Desai YM, Shah AH, Popplewell N. Three-degree-of-freedom model for galloping. Part I: Formulation; Part II: Solutions. J Eng Mech, ASCE 1993;119(12):2404-48.

[8] McConnel KG, Chang CN. A study of the axial-torsional coupling effect on a sagged transmission line. Exp Mech 1986:324-9.

[9] White WN, Venkatasubramanian S, Lynch PM, Huang CD. The equations of motion for the torsional and bending vibrations of a stranded cable. Applied Mechanics Division, ASME Paper No. 91-WA/APM-19; 1991.

[10] Luongo A, Zulli D, Piccardo G. A linear curved-beam model for the analysis of galloping in suspended cables. J Mech Mater Struct 2007;2(4):675-94.

[11] Lu CL, Perkins NC. Nonlinear spatial equilibria and stability of cables under uni-axial torque and thrust. J Appl Mech 1994;61:879-86.

[12] Nayfeh AH, Mook DT. Nonlinear oscillations. New York: John Wiley; 1979.

[13] Luongo A, Zulli D, Piccardo G. A nonlinear model of a curved beam for the analysis of galloping of suspended cables. In: Topping BHV, Montero G, Montenegro R, editors. Proceedings of the eighth international conference on computational structures technology, Civil-Comp Press, Stirlingshire, UK, Paper 93, 2006.

[14] Meirovitch L. Principles and techniques of vibrations. Prentice-Hall International Inc.; 1997.

[15] Irvine HM, Caughey TK. The linear theory of free vibrations of a suspended cable. Proc Royal Soc London, Ser A 1974;341:299-315.

[16] Wolfram S. The Mathematica book. Cambridge University Press; 2005.

[17] Nayfeh AH, Chin CM. Perturbation methods with Mathematica. Blacksburg (VA): Dynamic Press Inc.; 1999.

[18] Luongo A, Piccardo G. A continuous approach to the aeroelastic stability of suspended cables in $1: 2$ internal resonance. J Vib Control 2008;14(12):135-57.

[19] Hong KJ, Der Kiureghian A, Sackman JL. Bending behavior of helically wrapped cables. J Eng Mech, ASCE 2005;131(5):500-11.

[20] Tunstall M. Accretion of ice and aerodynamic coefficients. In: Proceedings of the Association des Ingénieurs Montefiore AIM, Study Day on Galloping, University of Liege, Liege, Belgium; 1989. 